\title{
An Axiomatic Approach To Systemic Risk
}

\author{
Chen Chen \\ Industrial Engineering and Operations Research \\ Columbia University \\ email: cc3136@columbia.edu \\ Garud lyengar \\ Industrial Engineering and Operations Research \\ Columbia University \\ email: garud@ieor.columbia.edu \\ Ciamac C. Moallemi \\ Graduate School of Business \\ Columbia University \\ email: ciamac@gsb.columbia.edu
}

Initial Version: August 4, 2011

Current Revision: April 18, 2012

\begin{abstract}
Systemic risk refers to the risk of collapse of an entire complex system, as a result of the actions taken by the individual component entities or agents that comprise the system. Systemic risk is an issue of great concern in modern financial markets as well as, more broadly, in the management of complex business and engineering systems. We propose an axiomatic framework for the measurement and management of systemic risk based on the simultaneous analysis of outcomes across agents in the system and over scenarios of nature. Our framework defines a broad class of systemic risk measures that accomodate a rich set of regulatory preferences. This general class of systemic risk measures captures many specific measures of systemic risk that have recently been proposed as special cases, and highlights their implicit assumptions. Moreover, the systemic risk measures that satisfy our conditions yield decentralized decompositions, i.e., the systemic risk can be decomposed into risk due to individual agents. Furthermore, one can associate a shadow price for systemic risk to each agent that correctly accounts for the externalities of the agent's individual decision-making on the entire system.
\end{abstract}

\section{Introduction}

The measurement and management of systemic risk is of fundamental importance in many business and engineering domains. The manager of a diversified firm has to assess and control the collective risk of all individual divisions or business units. The manager of a supply chain network is interested in the overall risk associated with a complex network of suppliers and sub-contractors. The manager of an electric power distribution network is interested in the aggregate risk of the generating stations, transmission facilities, and other entities in the network. As highlighted by the financial crisis of 2007-2008, one example of particular interest is the measurement and regulation of systemic risk of an economy or a financial market. While our methods are general and we seek to develop an understanding of systemic risk management broadly, in this paper, we focus on this last case and use the language of financial markets to present our work. 
Fundamentally, the study of systemic risk in a financial market involves the simultaneous analysis of outcomes across all entities (firms) in the economy. On the other hand, much of the academic literature on the theoretical foundations for the measurement and estimation of risk, as well as the main regulatory standards for risk, have been focused on the study of individual firms in isolation. We seek to bridge this gap by developing an axiomatic framework for a broad class of systemic risk measures.

Specifically, we are interested in an approach to systemic risk that is based on the analysis of the joint distribution of profits and losses across all firms in the economy and states of nature. We consider systemic risk from the perspective of a regulator, who wishes to express a preference over sets of possible distributions of outcomes for the entire economy. One approach to defining a systemic risk measure is to apply a traditional, single-firm risk measure such as value-at-risk or conditional value-at-risk to the distribution of the total profits and losses for all firms in the economy (e.g., Adrian and Brunnermeier, 2009: Acharya et al., 2010a; Tarashev et al., 2010). This approach treats the entire economy as a portfolio consisting of the constituent firms, and the regulator as a portfolio manager. However, the portfolio approach suffers from a number of modeling shortcomings. It implicitly allows the netting of profits and losses across the portfolio components. This is reasonable from the perspective of the manager of an investment portfolio. However, such netting may be undesirable from the perspective of a systemic regulator who, typically, is not able to directly cross-subsidize different firms with distinct ownership interests. Moreover, by considering only the total outcome, the portfolio approach lacks the modeling flexibility to accommodate preferences over the cross-sectional distribution of outcomes in an economy. For example, the regulator may have views on whether it is preferable for a single firm to have a large loss or many firms to have small losses, or whether profits at one firm can subsidize losses at another.

Motivated by these concerns, we define a broad class of systemic risk measures that can accommodate a rich set of regulatory preferences. The main contributions of this paper are as follows:

- We define an axiomatic framework defining systemic risk, and establish an associated structural decomposition.

Our work parallels the axiomatic approach to single-firm risk measures introduced by Artzner et al. (1999). Schied (2006) provides a very good survey of the extensive literature on coherent and convex risk measures for a single firm. Unlike the single-firm case, however, we consider a system or economy that consists of multiple components or firms. Systemic risk is then defined functional on the joint distribution of outcomes across firms in an economy and scenarios (states of nature) that satisfies a set of axioms. While we impose many axioms developed originally for single-firm risk measures to systemic risk measures to address similar concerns; we introduce two new axioms that are new. In particular, as in the case of coherent risk measures for a single firm, we assume the monotonicity and positive homogeneity of systemic risk. Besides the usual notion of convexity, we introduce a new risk convexity concept for situations where outcomes are not directly combined. Additionally, we assume a preference consistency condition that relates to the interactions between different firms across 
scenarios. The latter condition is novel and fundamentally specific to systemic risk; it has no analog among the typical conditions for single-firm risk measures, and it becomes trivial if the economy consists of a single firm.

We demonstrate that any systemic risk measure satisfying our definition can be characterized by two independent components: (1) an aggregation function that expresses a preference over the cross-sectional profile of outcomes across firms in a single scenario, and (2) a base risk measure, similar to existing single-firm risk measures, that expresses a preference over the profile of aggregated outcomes across scenarios of nature. This structural decomposition provides a clear structural characterization of systemic risk, and suggests a well-defined procedure to construct such risk measures by choosing constituent aggregation functions and base risk measures. This decomposition highlights the power of the preference consistency condition.

Our framework includes many recently proposed systemic risk measures as special cases. For example, a number of authors analyze systemic risk by applying single-firm risk measures to a portfolio consisting of all firms in the economy (e.g., Gauthier et al., 2010, Tarashev et al. 2010). The 'systemic expected shortfall' risk measure of Acharya et al. (2010a) employs a portfolio approach, with an expected shortfall base risk measure. An alternative reducedform approach to systemic risk involves considering the price of deposit insurance or other credit insurance (e.g., Lehar, 2005, Huang et al., 2009). Giesecke and Kim (2011) consider a risk measure defined through the fraction of failed firms in the economy. The general framework in this paper subsumes a number of these approaches. In Section 2, we illustrate how portfolio-based approaches to systemic risk measures such as the systemic expected shortfall or deposit insurance can be modeled as special cases in our framework. However, our framework provides greater flexibility in modeling systemic risk, allowing, for example, complex non-linear interactions between firms.

- We establish a dual representation for systemic risk, that allows attribution of risk to individual agents.

We show that any systemic risk measure can be expressed as the worst-case expected loss over a family of distributions over scenarios of nature and the cross-sectional profiles of firms, a generalization of the dual representation for single-firm coherent risk measures (Artzner et al., 1999). In many cases, this representation provides operational benefits by permitting decentralized computation of systemic risk by the firms in the economy. Moreover, we show that the dual variables are, in fact, shadow prices for systemic risk: they represent the marginal increase in systemic risk as a function of a marginal increase in the loss of a particular firm in a particular scenario.

In our setting, the dual representation provides a mechanism for risk attribution. The total systemic risk can always be apportioned across the constituent firms in a way that satisfies a 'no-undercut' condition: the systemic risk allocated to any subset of the firms is no more 
than the systemic risk those firms would face as a stand-alone economy. Our allocation rule is a generalization of the Aumann-Shapley prices for fair allocation of costs or the Euler allocation rule for allocating the capital requirements of a portfolio across constituent subportfolios (Denault, 2001; Buch and Dorfleitner, 2008). Similarly, the 'marginal expected shortfall' risk attribution of Acharya et al. (2010a) is a special case of our attribution rule.

We show that the risk attribution can properly account for the externalities imposed on the system when making decisions involving risk. Specifically, through a decentralized taxation scheme, the objective of the regulator can be aligned with the incentives of individual firms. Here, each individual firm maximizes the difference between its individual utility function and a tax payment that is derived from the firm's contribution to the systemic risk.

- Our methodology extends to a general class of risk measures. The structural decomposition of a systemic risk function into an aggregation function and a base risk measure follows from the preference consistency condition, and therefore, can be extended to broader classes of risk measures. In Section 5, we consider homogeneous systemic risk measures. These are systemic risk measures that are positively homogeneous and monotonic, but not necessarily convex. One example of such a risk measure is that of Adrian and Brunnermeier (2009), who define a risk measure based on the value-at-risk of the economy-wide portfolio. We show that homogeneous systemic risk measures that satisfy preference consistency can be decomposed into a single-firm homogeneous base risk measure and a homogeneous aggregation function. We describe a risk attribution scheme for a special class of piecewise linear homogeneous systemic risk measures that is a generalization of Aumann-Shapley prices. Similarly, a convex, monotonic, but not necessarily positively homogeneous, systemic risk measure that satisfies preference consistency can also be decomposed into a convex monotonic single-firm risk measure and a convex monotonic aggregation function.

Other authors have sought to model the structural mechanisms of interaction between firms in a financial crisis. Such models explicitly describe the contagion of credit events across firms in an economy through different structural mechanisms. For example, Acharya et al. (2010b) and Staum (2011a) consider asset price contagion, while Eisenberg and Noe (2001), Liu and Staum (2010), and Cont et al. (2010) consider counterparty contagion. Staum (2011b) provides an excellent survey of the literature on contagion and systemic risk. In this paper, we take as given a collection of exogenous outcomes across firms and scenarios of nature. However, we can accomodate aspects of endogenous, structural mechanisms for contagion through the choice of risk measure. This is illustrated in Example 7 in Section 2.

The rest of the paper is organized as follows: In Section 2, we provide an axiomatic definition of a systemic risk measure. In Section 2.1, we describe the structural decomposition of systemic risk, and in Section 2.2 we discuss a number of examples of systemic risk measures. In Section 3 . we construct primal and dual variational representations for systemic risk measures. In Section 4. we discuss a systemic risk attribution scheme. In Section 5, we present extensions of our theory to 
homogeneous systemic risk measures. In the Online Supplement, we demonstrate a decentralized framework for systemic risk management, as well as provide proofs.

\section{Model}

We consider a one-period model consisting of a finite set of firms $\mathcal{F}$ and a finite set of future scenarios $\Omega$. We define an economy by a matrix $X \in \mathbb{R}^{|\mathcal{F}| \times|\Omega|}$. Here, the quantity $X_{i, \omega}$ is the loss (or, if negative, the profit) of firm $i$ in scenario $\omega$. We denote by $X_{\omega} \in \mathbb{R}^{|\mathcal{F}|}$ the column vector of outcomes in scenario $\omega$ across all firms; we refer to this as the cross-sectional profile of losses across firms of the economy $X$, in scenario $\omega$. In some examples, we assume there is a probability distribution $p \in \mathbb{R}_{+}^{|\Omega|}$ over the space of scenarios $\Omega$. In these cases, we can interprete the matrix $X \in \mathbb{R}^{|\mathcal{F}| \times|\Omega|}$ as a random vector which has outcome $X_{\omega} \in \mathbb{R}^{|\mathcal{F}|}$ with probability $p_{\omega}$.

In the rest of the paper, the following notation is helpful: the vector $\mathbf{1}_{\Omega} \in \mathbb{R}^{|\Omega|}$ denotes a unit loss of an individual firm in all scenarios, and vector $\mathbf{1}_{\mathcal{F}} \in \mathbb{R}^{|\mathcal{F}|}$ denotes a cross-sectional loss profile in a scenario where each firm has a unit loss, and the matrix $\mathbf{1}_{\mathcal{E}} \triangleq \mathbf{1}_{\mathcal{F}} \mathbf{1}_{\Omega}^{\top} \in \mathbb{R}^{|\mathcal{F}| \times|\Omega|}$ denotes an economy with a unit loss for every firm in every scenario. Similarly, the vectors $\mathbf{0}_{\mathcal{F}} \in \mathbb{R}^{|\mathcal{F}|}$, $\mathbf{0}_{\Omega} \in \mathbb{R}^{|\Omega|}$, and the matrix $\mathbf{0}_{\mathcal{E}} \in \mathbb{R}^{|\mathcal{F}| \times|\Omega|}$ correspond to cases with zero profit or loss for every firm in every scenario. Given an economy $X$, a cross-sectional loss profile $x$, and a scenario $\omega$, the matrix $\left(x, X_{-\omega}\right) \in \mathbb{R}^{|\mathcal{F}| \times|\Omega|}$ denotes an economy with loss profile $x$ in scenario $\omega$, but where outcomes in all other scenarios are given by the corresponding columns in $X$. Inequalities between pairs of vectors and matrices are to be interpreted component-wise.

A systemic risk measure $\rho$ is a summary statistic that quantifies the level of 'risk' associated with an economy $X$ by a single real number $\rho(X)$. Given two economies $X$ and $Y$, if $\rho(X)>\rho(Y)$ then we say that $X$ is riskier than $Y$ and thus less preferred. Hence, a systemic risk measure implicitly encodes the preferences of a regulator over the universe of possible economies.

We first review the axiomatic framework for coherent single-firm ${ }^{1}$ risk measures ${ }^{2}$ commonly used in the literature (Artzner et al., 1999).

Definition 1 (Single-Firm Risk Measure). A single-firm risk measure is a function ${ }^{3}: \mathbb{R}^{|\Omega|} \rightarrow \mathbb{R}$ that satisfies the following conditions $(i)($ iv $)$, for all loss vectors $x, y \in \mathbb{R}^{|\Omega|}$ of a single firm:

(i) Monotonicity: If $x \geq y$, then $\rho(x) \geq \rho(y)$.

(ii) Positive homogeneity: For all non-negative scalars $\alpha \geq 0, \rho(\alpha x)=\alpha \rho(x)$.

\footnotetext{
${ }^{1}$ In this paper, we use the term 'single-firm' risk measure to refer to the risk measures for a single entity, i.e., an entity for which the outcome in every scenario of nature is a single real number. This is in contrast to the systemic risk measures which we will introduce shortly, where there is a vector of outcomes (one for each component of the system) in every scenario of nature. Note that a systemic risk measure could, for example, also be applied in the case of an individual firm, where the 'components' correspond to divisions of the firm that contribute to the overall risk.

${ }^{2}$ Our terminology is slightly non-standard here: for example, for single-firm risk measures, Schied (2006) defines a 'monetary measure of risk' as satisfying (i) and (v)] a 'convex measure of risk' as satisfying (i) and (iii) (v) and a 'coherent measure of risk as satisfying (i) (v).

${ }^{3}$ In what follows, we sometimes consider single-firm risk measures $\rho: \mathbb{R}_{+}^{|\Omega|} \rightarrow \mathbb{R}$ defined only on the positive orthant. In that case, we assume that conditions (i) (iv) are satisfied for all $x, y \in \mathbb{R}_{+}^{|\Omega|}$.
} 
(iii) Convexity: Given a scalar $0 \leq \alpha \leq 1, \rho(\alpha x+(1-\alpha y)) \leq \alpha \rho(x)+(1-\alpha) \rho(y)$.

(iv) Normalization: $\rho\left(\mathbf{1}_{\Omega}\right)=1$.

If in addition, a single-firm risk measure satisfies the following condition (v), it is called coherent:

(v) Cash invariance: For all scalars $\alpha \in \mathbb{R}, \rho\left(x+\alpha \mathbf{1}_{\Omega}\right)=\rho(x)+\alpha$.

The conditions for a single-firm risk measure can be motivated as follows: The monotonicity condition (i) reflects that, if one firm has greater losses in every scenario than another, it is less preferred. The positive homogeneity condition (ii) requires that the risk increases in proportion to the scale of losses. The convexity condition (iii) asserts that diversification reduces risk, i.e., the risk of a firm diversified between outcomes corresponding to $x$ and $y$ is less than the weighted risk of the component firms $x$ and $y$. The normalization condition (iv) fixes the multiplicative scaling ${ }^{4}$ of the risk measure. The cash invariance condition (v) allows the interpretation of risk as a capital requirement: when a certain loss $\alpha$ is added to the outcome in every scenario, the risk of the firm increases by exactly $\alpha$.

Building on the definition for a single-firm risk measure, we formally define a systemic risk measure as follows:

Definition 2 (Systemic Risk Measure). A systemic risk measure is a function $\rho: \mathbb{R}^{|\mathcal{F}| \times|\Omega|} \rightarrow \mathbb{R}$ that satisfies the following conditions, for all economies $X, Y, Z \in \mathbb{R}^{|\mathcal{F}| \times|\Omega|}$ :

(I) Monotonicity: If $X \geq Y$, then $\rho(X) \geq \rho(Y)$.

(II) Positive homogeneity: For all non-negative scalars $\alpha \geq 0, \rho(\alpha X)=\alpha \rho(X)$.

(III) Preference consistency: Define a partial order $\succeq_{\rho}$ on cross-sectional profiles as follows: Given cross-sectional profiles $x, y \in \mathbb{R}^{|\mathcal{F}|}$, we say that $x \succeq_{\rho}$ y iff $\rho\left(x \mathbf{1}_{\Omega}^{\top}\right) \geq \rho\left(y \mathbf{1}_{\Omega}^{\top}\right)$. Suppose that, for every scenario $\omega, X_{\omega} \succeq_{\rho} Y_{\omega}$. Then, $\rho(X) \geq \rho(Y)$.

(IV) Convexity:

(a) Outcome convexity: Suppose $Z=\alpha X+(1-\alpha) Y$, for a given scalar $0 \leq \alpha \leq 1$. Then, $\rho(Z) \leq \alpha \rho(X)+(1-\alpha) \rho(Y)$.

(b) Risk convexity: Suppose $\rho\left(Z_{\omega} \mathbf{1}_{\Omega}^{\top}\right)=\alpha \rho\left(X_{\omega} \mathbf{1}_{\Omega}^{\top}\right)+(1-\alpha) \rho\left(Y_{\omega} \mathbf{1}_{\Omega}^{\top}\right), \forall \omega \in \Omega$, for a given scalar $0 \leq \alpha \leq 1$. Then, $\rho(Z) \leq \alpha \rho(X)+(1-\alpha) \rho(Y)$.

(V) Normalization: $\rho\left(\mathbf{1}_{\mathcal{E}}\right)=|\mathcal{F}|$.

Our definition of a systemic risk measure is justified as follows: conditions (I) (II) are similar to the corresponding conditions for a single-firm risk measure, and can be justified in a similar

\footnotetext{
${ }^{4}$ Note that in the case of a coherent single-firm risk measure, the normalization condition (iv) follows from positive homogeneity and cash invariance. In our work, it will be useful to consider non-coherent risk measures, hence we retain this as a separate condition.
} 
manner. The preference consistency condition (III), on the other hand, does not have an analog in the single-firm case. This condition defines an ordering (or, preference relationship) $\succeq_{\rho}$ on cross-sectional profiles $x, y \in \mathbb{R}^{|\mathcal{F}|}$ by comparing the systemic risk (according to $\rho$ ) of the constant economies $^{5} x \mathbf{1}_{\Omega}^{\top}$ and $y \mathbf{1}_{\Omega}^{\top}$. If $x \succeq_{\rho} y$, we say that $y$ is preferred to $x$. Preference consistency requires that if cross-sectional profiles in the economies $X$ and $Y$ are such that if, in every scenario $\omega, Y_{\omega}$ is preferred to $X_{\omega}$, the systemic risk of $Y$ must be consistent with this preference and thus cannot be greater than the systemic risk of $X$. When the economy consists of a single firm, condition (III) follows from monotonicity.

The preference consistency condition implies independence from irrelevant alternatives (see, e.g., Kreps, 1988) as follows: suppose that $x, y \in \mathbb{R}^{|\mathcal{F}|}$ are cross-sectional loss profiles such that $x \succeq_{\rho} y$, i.e., $y$ is preferred to $x$. Then, for any economy $Z \in \mathbb{R}^{|\mathcal{F}| \times|\Omega|}$ and any scenario $\omega$, define $\left(x, Z_{-}\right)$to be the economy where outcomes for firms in scenario $\omega$ are given by $x$, and outcomes in all other scenarios are given by $Z$, and define $\left(y, Z_{-\omega}\right)$ similarly. Preference consistency implies that $\rho\left(x, Z_{-\omega}\right) \geq \rho\left(y, Z_{-\omega}\right)$. In other words, if $y$ is preferred to $x$, then, all else being equal, any economy which realizes $y$ in some scenario is less risky to an economy which realizes $x$ in that scenario, independent of the scenario and of the outcomes in other scenarios. Thus, by imposing the preference consistency axiom, we assume that the systemic risk measure expresses a preference over cross-sectional profiles that is consistent across scenarios. Introducing the preference consistency condition is one of the major contributions of this paper, in that it allows us to extend the single-firm risk measure to a systemic risk measure that captures the interaction of many firms.

The convexity conditions (IV-a) and (IV-b) are both concerned with the benefits of diversification. Condition (IV-a), labeled 'outcome convexity', is the usual notion of convexity: when the economy $Z$ is a diversified mixture of two economies $X$ and $Y$, the risk of $Z$ is no greater than the weighted combination of the risk of economies $X$ and $Y$. Outcome convexity is concerned with a 'portfolio' of economies $X$ and $Y$, in that we are allowed to add the outcomes from the two economies and the risk reduction comes from the fact that outcomes $X$ and $Y$ are possibly correlated.

Condition (IV-b) is concerned with convexity as it relates to risk aversion. The context of this condition is as follows. We have two stages of uncertainty. The outcome of the first stage is the economy $X$ with probability $\alpha$ and the economy $Y$ with probability $1-\alpha$. In the second stage, the scenario $\omega$ and the firm outcomes corresponding to $\omega$ and the economy selected in the first stage are revealed. Note that in this setting we do not have a 'portfolio' of economies. The economy $Z$ in condition (IV-b) is such that in every scenario $\omega \in \Omega$ the risk $\rho\left(Z_{\omega} \mathbf{1}_{\Omega}^{\top}\right)$ is a convex combination of the risk $\rho\left(X_{\omega} \mathbf{1}_{\Omega}^{\top}\right)$ and $\rho\left(Y_{\omega} \mathbf{1}_{\Omega}^{\top}\right)$, i.e., the outcomes of economy $Z$ are not subject to the first stage randomness. Condition (IV-b) states that the risk of the 'average' economy $Z$ is at most the convex combination $\alpha \rho(X)+(1-\alpha) \rho(Y)$. The risk reduction in this case comes from the removing one stage of randomness. When the economy consists of a single firm, condition (IV-b) is implied by

\footnotetext{
${ }^{5}$ Note that, given $x \in \mathbb{R}^{|\mathcal{F}|}$, the economy $x \mathbf{1}_{\Omega}^{\top}$ has the same cross-sectional profile of losses $x$ in every scenario. Hence, we call this a constant economy.
} 
cash invariance and outcome convexity.

Finally, the normalization condition $(\mathrm{V})$ requires the risk of a unit loss by all firms with certainty to be the total loss, i.e., the number of firms $|\mathcal{F}|$. This is simply a convenient choice of scaling and is imposed without loss of generality.

Note that our definition of systemic risk does not contain a cash invariance condition, as required by a coherent single-firm risk measure. This is because we want to allow for systemic risk measures derived from deposit insurance that are incompatibile with cash invariance: if all outcomes in the future are reduced by a deterministic amount, this does not necessarily result in a commensurate reduction in the price of deposit insurance.

\subsection{Structural Decomposition}

In order to assess the systemic risk of an economy, a regulator is concerned with both the crosssectional profile of losses across firms and the distribution of aggregate outcomes across scenarios. Thus, in order to define a risk preference over the universe of economies, one might seek to independently express these two types of preferences. We formalize this notion as follows:

Definition 3 (Aggregation Function). A function $\Lambda: \mathbb{R}^{|\mathcal{F}|} \rightarrow \mathbb{R}$ over cross-sectional loss profiles of firms is an aggregation function if, for all cross-sectional loss profiles $x, y \in \mathbb{R}^{|\mathcal{F}|}$, it satisfies:

(i) Monotonicity: If $x \geq y$, then $\Lambda(x) \geq \Lambda(y)$.

(ii) Positive homogeneity: For all $\alpha \geq 0, \Lambda(\alpha x)=\alpha \Lambda(x)$.

(iii) Convexity: For all $0 \leq \alpha \leq 1, \Lambda(\alpha x+(1-\alpha y)) \leq \alpha \Lambda(x)+(1-\alpha) \Lambda(y)$.

(iv) Normalization: $\Lambda\left(\mathbf{1}_{\mathcal{F}}\right)=|\mathcal{F}|$.

An aggregation function provides a summary statistic that encapsulates a cross-sectional profile of losses across firms in a single scenario into a real number, thus expressing a preference over such profiles. The conditions (i) (iv) are analogous to the corresponding conditions for a systemic risk measure, and motivated by similar concerns. Subject to these conditions, the regulator has considerable freedom in specifying preferences over the distribution of losses across firms, and we will see a number of examples of aggregation functions in what follows.

Once the cross-sectional outcomes across firms are aggregated, the evaluation of systemic risk reduces to an evaluation of the profile of aggregated outcomes across scenarios. This can be accomplished by a single-firm risk measure $\rho_{0}: \mathbb{R}^{|\Omega|} \rightarrow \mathbb{R}$ (Definition 1 ), which we call the base risk measure. The independent choice of an aggregation function and a base risk measure provides a clear way to specify preferences over the universe of economies. The following theorem, whose proof is deferred until Section 2.3 , illustrates how these functions can be composed to yield a systemic risk measure, and that, in fact, all systemic risk measures admit such a decomposition.

Theorem 1. (i) A function $\rho: \mathbb{R}^{|\mathcal{F}| \times|\Omega|} \rightarrow \mathbb{R}$ is a systemic risk measure with image $\operatorname{Im} \rho=\mathbb{R}$ if and only if there exist an aggregation function $\Lambda: \mathbb{R}^{|\mathcal{F}|} \rightarrow \mathbb{R}$ with image $\operatorname{Im} \Lambda=\mathbb{R}$ and a 
coherent single-firm risk measure $\rho_{0}: \mathbb{R}^{|\Omega|} \rightarrow \mathbb{R}$, such that $\rho$ is the composition of $\rho_{0}$ and $\Lambda$, i.e.,

$$
\rho(X)=\left(\rho_{0} \circ \Lambda\right)(X) \triangleq \rho_{0}\left(\Lambda\left(X_{1}\right), \Lambda\left(X_{2}\right), \ldots, \Lambda\left(X_{|\Omega|}\right)\right), \quad \forall X \in \mathbb{R}^{|\mathcal{F}| \times|\Omega|}
$$

(ii) A function $\rho: \mathbb{R}^{|\mathcal{F}| \times|\Omega|} \rightarrow \mathbb{R}$ is a systemic risk measure with image $\operatorname{Im} \rho=\mathbb{R}_{+}$if and only if there exist an aggregation function $\Lambda: \mathbb{R}^{|\mathcal{F}|} \rightarrow \mathbb{R}$ with image $\operatorname{Im} \Lambda=\mathbb{R}_{+}$and a single-firm risk measure $\rho_{0}: \mathbb{R}_{+}^{\Omega} \rightarrow \mathbb{R}$, such that $\rho$ is the composition of $\rho_{0}$ and $\Lambda$, i.e.,

$$
\rho(X)=\left(\rho_{0} \circ \Lambda\right)(X) \triangleq \rho_{0}\left(\Lambda\left(X_{1}\right), \Lambda\left(X_{2}\right), \ldots, \Lambda\left(X_{|\Omega|}\right)\right), \quad \forall X \in \mathbb{R}^{|\mathcal{F}| \times|\Omega|}
$$

Note that for a systemic risk measure $\rho$ the positive homogeneity and the normalization conditions imply that $\operatorname{Im} \rho$ is either $\mathbb{R}$ or $\mathbb{R}_{+}$. Hence, the two parts in Theorem 1 state that, in all cases, the choice of a systemic risk measure is equivalent to the choice of a base risk measure and an aggregation function. Further, Theorem 1 does not guarantee the uniqueness of the base risk measure and aggregation function corresponding to a particular systemic risk measure.

As shown in Theorem 1, the key ingredient that bridges single-firm risk measures to systemic risk measures is the choice of aggregation function. An aggregation function allows us to measure the risk of aggregate positions as that of a single firm's positions. We emphasize that it is the preference consistency condition in Definition 2 that makes this structural decomposition possible. In fact, when the other conditions (including monotonicity, positive homogeneity, and convexity) are modified, a similar structural decomposition result continues to hold so long as preference consistency is satisfied. For example, in Section 5, we provide a structural decomposition when the convexity condition is dropped and the positive homogeneity condition is kept; similarly, a structural decomposition can also be constructed if the positive homogeneity condition is dropped and the convexity condition is kept. Simply put, the preference consistency condition connects a reasonable single-firm risk measure to a systemic version, and one has the freedom to choose other appropriate conditions for risk measure.

\subsection{Applications}

We now consider some examples to illustrate how the choice of an aggregation function and a base risk measure describes a systemic risk measure.

\section{Example 1 (Systemic Expected Shortfall). Consider the aggregation function}

$$
\Lambda_{\text {total }}(x) \triangleq \sum_{i \in \mathcal{F}} x_{i}=\mathbf{1}_{\mathcal{F}}^{\top} x, \quad \forall x \in \mathbb{R}^{|\mathcal{F}|}
$$

This aggregation function defines the aggregate loss of a cross-sectional profile to be the sum of the profits and losses of individual firms. Assume there is a given distribution $p \in \mathbb{R}_{+}^{|\Omega|}$ over the space 
of scenarios $\Omega$, and define

$$
\begin{aligned}
\mathrm{CVaR}_{\zeta}(y) \triangleq \underset{q \in \mathbb{R}^{|\Omega|}}{\operatorname{maximize}} & q^{\top} y \\
\text { subject to } & \mathbf{0}_{\Omega} \leq q \leq p / \zeta \\
& \mathbf{1}_{\Omega}^{\top} q=1
\end{aligned}
$$

for all $y \in \mathbb{R}^{|\Omega|}$. Here, $\operatorname{CVaR}_{\zeta}(y)$ is the expected shortfall or conditional value at risk of the $\zeta$ percentile of the aggregate loss vector $y$, where $0<\zeta<1$. By taking this as the base risk measure, we can define the systemic risk measure

$$
\rho_{S E S}(X) \triangleq \operatorname{CVaR}_{\zeta}\left(\mathbf{1}_{\mathcal{F}}^{\top} X_{1}, \ldots, \mathbf{1}_{\mathcal{F}}^{\top} X_{|\Omega|}\right), \quad \forall X \in \mathbb{R}^{|\mathcal{F}| \times|\Omega|} .
$$

This systemic risk measure is closely related ${ }^{6}$ to the 'systemic expected shortfall' of the economy discussed by Acharya et al. (2010a). Note that this choice of aggregation function treats losses and gains symmetrically. Further, it allows gains from one firm to cancel with losses of another firm. This approach might be undesirable if the regulator cannot subsidize the losses of some firms with the profits of others.

Example 2 (Deposit Insurance). Consider the aggregation function ${ }^{7}$

$$
\Lambda_{\text {loss }}(x) \triangleq \sum_{i \in \mathcal{F}} x_{i}^{+}, \quad \forall x \in \mathbb{R}^{|\mathcal{F}|}
$$

This aggregation function considers only the losses of the firms. Assume there is a given distribution $p \in \mathbb{R}_{+}^{|\Omega|}$ over the space of scenarios $\Omega$, and define the base risk measure to be the expectation

$$
\mathrm{E}[y]=p^{\top} y, \quad \forall y \in \mathbb{R}^{|\Omega|}
$$

Then, we have

$$
\rho_{D I}(X)=\mathrm{E}\left[\sum_{i \in \mathcal{F}} X_{i, \omega}^{+}\right], \quad \forall X \in \mathbb{R}^{|\mathcal{F}| \times|\Omega|} .
$$

In this example, the risk measure is the expected value of total losses only. When the expectation is taken over the risk neutral distribution, $\rho(X)$ equals the price of a 'deposit insurance' contract that pays out the losses of insolvent firms. This is similar in spirit to a number of proposed systemic risk measures (Lehar, 2005: Huang et al., 2009). Note that the aggregation function $\Lambda_{\text {loss }}$ treats losses and gains asymmetrically, and does not allow the gains of some firms to subsidize losses of other firms.

One feature common to both Examples 1 and 2 is that they are indifferent to how a large

\footnotetext{
${ }^{6}$ Strictly speaking, Acharya et al. (2010a) define a risk measure via preferences over the collection of returns of individual firms, while we express preferences over the losses, in absolute terms, experienced by individual firms. This difference is minor, however, and our risk measures could easily be defined in that setting.

${ }^{7}$ Given a scalar $z \in \mathbb{R}$, we define $z^{+} \triangleq \max (z, 0)$ to be the positive part of $z$, and $z^{-} \triangleq \max (-z, 0)$ to be the negative part of $z$.
} 
loss is spread out across firms in an economy. In particular, the aggregation functions $\Lambda_{\text {total }}$ and $\Lambda_{\text {loss }}$ assign the same aggregate outcome to a cross-sectional profile where one firm losses a lot of money and other firms have zero loss, or a profile where all firms lose an average amount of money. In practice, a regulator may have a preference over two such profiles. Through the design of appropriate aggregation functions, our framework is sufficiently rich to express such preferences.

Example 3 (Investing with Performance Fees). Consider an investor with investments in a collection of hedge funds indexed by the set $\mathcal{F}$. Here, given a loss profile $x \in \mathbb{R}^{|\mathcal{F}|}$, we interpret $x_{i}$ as the gross loss incurred by the investor due to the investment in the hedge fund $i$. Consider the following two cases: (a) the investor is a direct investor in the individual hedge funds; (b) the investor is indirectly invested in the individual hedge funds via a fund-of-funds. We assume that each hedge fund $i$ charges a performance fee that is a fraction $\gamma_{i} \in[0,1]$ of the gross profits (if any) generated by the fund for the investor. The fund-of-funds charges a performance fee that is a fraction $\gamma \in[0,1]$ of the aggregate profits (if any) of the investor across all of the funds, net of performance fees paid to the individual funds. In case (a), the direct hedge fund investor can express preferences over loss profiles via the aggregation function

$$
\Lambda_{\mathrm{HF}}(x) \triangleq \sum_{i \in \mathcal{F}}\left(x_{i}+\gamma_{i} x_{i}^{-}\right), \quad \forall x \in \mathbb{R}^{|\mathcal{F}|}
$$

In case (b), the fund-of-funds investor can do so via the aggregation function

$$
\Lambda_{\mathrm{FoF}}(x) \triangleq \sum_{i \in \mathcal{F}}\left(x_{i}+\gamma_{i} x_{i}^{-}\right)+\gamma\left(\sum_{i \in \mathcal{F}}\left(x_{i}+\gamma_{i} x_{i}^{-}\right)\right)^{-}, \quad \forall x \in \mathbb{R}^{|\mathcal{F}|} .
$$

These aggregation functions consider the total profit or loss across all funds to the investor, net of all performance fees.

In the above example, we measure the systemic risk from a portfolio management viewpoint of an investor. Here, because of performance fees, losses and gains must be treated asymmetrically. Moreover, dispersion risk is important: holding the gross profit $\sum_{i \in \mathcal{F}} x_{i}$ fixed, the investor prefers to eschew profiles where the individual fund outcomes $\left\{x_{i}\right\}$ are dispersed, and the investor pays fees to the positively performing funds, but does not recover fees from the negatively performing funds. With modifications, more complicated performance fee structures or tax schemes imposed on profits can be captured by a similar aggregation functions. In these examples, the choice of the base risk measure is left up to the investor.

In the following examples, we illustrate systemic risk measures that are not restricted to financial applications. For complex systems with many interacting components, we can often design systemic risk measures with specialized structure appropriate for the application at hand. The examples we consider involve aggregation functions that are special cases of the following general class:

Example 4 (Optimization Aggregation Function). Given matrices $A \in \mathbb{R}_{+}^{K \times|\mathcal{F}|}, B \in \mathbb{R}^{K \times N}$, a vector 
$c \in \mathbb{R}_{+}^{N}$, and a convex cone $\mathcal{K} \subset \mathbb{R}^{N}$, define $\Lambda_{\mathrm{OPT}}: \mathbb{R}^{|\mathcal{F}|} \rightarrow \mathbb{R}$ by

$$
\begin{aligned}
\Lambda_{\mathrm{OPT}}(x) \triangleq & \underset{y \in \mathcal{K}}{\operatorname{minimize}} \quad c^{\top} y \\
\text { subject to } & A x \leq B y .
\end{aligned}
$$

for all loss profiles $x \in \mathbb{R}^{|\mathcal{F}|}$. If we assume that, for example,

$$
\exists \bar{y} \in \mathcal{K} \text { such that } B \bar{y}>\mathbf{0}, \quad \exists \bar{z} \in \mathbb{R}_{+}^{K} \text { such that } B^{\top} \bar{z}=c,
$$

then it is not difficult to see that the program (6) is feasible and bounded for all $x$, and that $\Lambda_{\mathrm{OPT}}$ is monotonic, positively homogeneous, and convex. Further, if $\Lambda_{\mathrm{OPT}}\left(\mathbf{1}_{\mathcal{F}}\right)>0$, c can be rescaled such that $\Lambda_{\mathrm{OPT}}$ is normalized as well, and thus is an aggregation function.

Note that $\Lambda_{\text {OPT }}$ captures a broad class of aggregation functions, including all of the previous examples in this section. In the following examples, we illustrate that optimization aggregation functions can be naturally applied in practical settings, including many non-financial applications.

Example 5 (Resource Allocation). Consider a resource allocation setting, where $\mathcal{F}$ denotes a set of capacitated resources, and $\mathcal{A}$ denotes a collection of activities. Suppose activity a consumes resource $r \in \mathcal{F}$ at rate $b_{r a} \geq 0$ per unit of activity. Given a loss profile $x \in \mathbb{R}^{|\mathcal{F}|}$, we interpret each loss $x_{r}$ as a shortage (or, if negative, the surplus) to the available supply of resource $r$, relative to a baseline utilized capacity. Define the aggregation function

$$
\begin{aligned}
\Lambda_{\mathrm{RA}}(x) \triangleq \operatorname{minimize}_{u \in \mathbb{R}^{|\mathcal{A}|}} & \sum_{a \in \mathcal{A}} c_{a} u_{a} \\
\text { subject to } & \sum_{a \in \mathcal{A}} b_{r a} u_{a} \geq x_{r}, \quad \forall r \in \mathcal{F} .
\end{aligned}
$$

Here, each decision variable $u_{a}$ is the reduction (or, if negative, the increase) of the level of activity $a$. The constraint enforces the requirement that consumption of each resource $r$ across activities be adjusted so as to accommodate the resource supply reduction $x_{r}$. The vector $c \geq \mathbf{0}$ specifies the cost associated with reductions in the level of each activity. Given a resource supply shock $x, \Lambda_{\mathrm{RA}}(x)$ measures the cost of the corresponding optimized reduction in activities. Hence, $\Lambda_{\mathrm{RA}}$ reflects the preferences of a system manager in a resource allocation setting.

Example 6 (Flow Network). Consider a network with vertices $\mathcal{V}$ and a set $\mathcal{F} \subset \mathcal{V} \times \mathcal{V}$ of directed edges. Each edge $(u, v) \in \mathcal{F}$ corresponds to a capacitated link, and the goal of the network manager is to direct maximal flow from a source $s \in \mathcal{V}$ to a destination $t \in \mathcal{V}$. Given a loss profile $x \in \mathbb{R}^{|\mathcal{F}|}$, we interpret each loss $x_{(u, v)}$ as a reduction (or, if negative, an increase) to the capacity of link 
$(u, v) \in \mathcal{F}$, relative to a baseline level of utilized capacity. Define the aggregation function

$$
\begin{array}{lll}
\Lambda_{\mathrm{NF}}(x) \triangleq{\underset{f \in \mathbb{R}}{|\mathcal{F}|}}_{\operatorname{minimize}} & \sum_{v:(s, v) \in \mathcal{F}} f_{(s, v)} & \\
\text { subject to } & f_{(u, v)} \geq x_{(u, v)}, \quad \forall(u, v) \in \mathcal{F}, \\
\sum_{u:(u, w) \in \mathcal{F}} f_{(u, w)} & =\sum_{v:(w, v) \in \mathcal{F}} f_{(w, v)}, \quad \forall w \in \mathcal{V} \backslash\{s, t\} .
\end{array}
$$

Here, each decision variable $f_{(u, v)}$ represents the required reduction of flow along the link $(u, v)$. The first constraint enforces the requirement that flows be reduced so as to accommodate the capacity shock $x$, while the second constraint is a flow balance equation. Given a capacity shock $x, \Lambda_{\mathrm{NF}}(x)$ measures the minimal necessary flow reduction, and hence reflects the preferences of a manager in a max-flow setting.

In previous examples, we have viewed outcomes across firms and scenarios of nature as exogenously specified and did not consider structural mechanisms by which the loss of one firm can create losses at other firms, i.e., contagion. The following example illustrates that it is possible to introduce mechanisms for contagion, through the careful definition of the value function.

Example 7 (Contagion Model $^{8}$ ). Let $\mathcal{F}$ denote a collection of firms, each of whom has certain assets and obligations to each other. Let $\Pi_{i j}$ denote the fraction of the total debt of firm $i$ that is owed to firm $j$. Let $x \in \mathbb{R}^{|\mathcal{F}|}$ denote the loss profile in a particular scenario. Define the aggregation function

$$
\begin{aligned}
& \Lambda_{\mathrm{CM}}(x) \triangleq \operatorname{minimize}_{y \in \mathbb{R}_{+}^{|\mathcal{F}|}, b \in \mathbb{R}_{+}^{|\mathcal{F}|}} \sum_{i \in \mathcal{F}} y_{i}+\gamma \sum_{i \in \mathcal{F}} b_{i} \\
& \text { subject to } \quad b_{i}+y_{i} \geq x_{i}+\sum_{j \in \mathcal{F}} \Pi_{j i} y_{j}, \quad \forall i \in \mathcal{F} \text {. }
\end{aligned}
$$

We interpret $x$ as losses external to the obligations the firms have to each other. The loss $x_{i}$ must be covered either by firm $i$ reducing the payments on its obligations to other firms by an amount $y_{i}$, or relying on an injection of external funds from the regulator in the amount $b_{i}$. The parameter $\gamma>1$ balances the preferences of the regulator in trading off between, on the one hand, the aggregate shortfalls across the economy on inter-firm obligations $\sum_{i \in \mathcal{F}} y_{i}$ and, on the other hand, the cost $\sum_{i \in \mathcal{F}} b_{i}$ of injecting new capital to support the economy. The feasibility constraints reflects the fact that the reduction in payment $y_{i}$ by firm $i$ results in an additional loss of an amount $\Pi_{i j} y_{i}$ for firm $j$. The aggregation function $\Lambda_{\mathrm{CM}}$ measures the net systemic cost of the contagion. It is monotonic, positively homogeneous, and convex. Also, it can be normalized since $\Lambda_{\mathrm{CM}}\left(\mathbf{1}_{\mathcal{F}}\right)>0$.

The contraint in (9) is inspired by the structural contagion model of Eisenberg and Noe (2001). However, in their model, the firms have limited liability, i.e., $y \leq \bar{p}$ for some vector $\bar{p} \in \mathbb{R}_{+}^{|\mathcal{F}|}$ of total liabilities, and there is no external injection of capital, i.e., $b \triangleq 0$. In that case, however, the aggregation function would not be positively homogeneous. Motivated by this example, an interesting

\footnotetext{
${ }^{8}$ We thank an anonymous reviewer for suggesting this example.
} 
possible extension of our work would be to consider such convex but not positively homogeneous systemic risk measures.

\subsection{Proof of Theorem 1}

The two parts in this theorem have essentially one proof with minor differences. First, suppose that $\rho$ is a systemic risk measure. For each loss profile $x \in \mathbb{R}^{|\mathcal{F}|}$, define

$$
\Lambda(x) \triangleq \rho\left(x \mathbf{1}_{\Omega}^{\top}\right)
$$

In other words, $\Lambda$ computes the systemic risk of a constant economy represented by $x$. The monotonicity, convexity and positive homogeneity of $\Lambda$ holds due to the monotonicity, convexity, and positive homogeneity of $\rho$. Also, $\Lambda\left(\mathbf{1}_{\mathcal{F}}\right)=|\mathcal{F}|$, since $\rho\left(\mathbf{1}_{\mathcal{E}}\right)=|\mathcal{F}|$. Let $\mathcal{Q} \triangleq \operatorname{Im}(\Lambda)$ be the image of $\Lambda$. We know $|\mathcal{F}| \in \mathcal{Q}$. By the positive homogeneity of $\Lambda$, we conclude that $\mathbb{R}_{+} \subset \mathcal{Q}$. Suppose there exists an economy $X$ such that $\rho(X)<0$. We can find a vector $x \in \mathbb{R}^{|\mathcal{F}|}$ such that $x \mathbf{1}_{\Omega}^{\top} \leq X$. So $\Lambda(x)=\rho\left(x \mathbf{1}_{\Omega}^{\top}\right) \leq \rho(X)<0$. By the positive homogeneity of $\Lambda$, we conclude that $\mathbb{R}_{-} \subset \mathcal{Q}$. Thus, for part (i), $\mathcal{Q}=\mathbb{R}$; for part (ii), $\mathcal{Q}=\mathbb{R}_{+}$. For each vector $z \in \mathcal{Q}^{\Omega}$, define

$$
\rho_{0}(z) \triangleq \rho(X)
$$

where $X$ is an economy that satisfies

$$
\Lambda\left(X_{\omega}\right)=z_{\omega}, \quad \forall \omega \in \Omega
$$

First, we show $\rho_{0}$ is well-defined. Suppose two economies $X, Y$ have that

$$
\Lambda\left(X_{\omega}\right)=\Lambda\left(Y_{\omega}\right), \quad \forall \omega \in \Omega
$$

Since $\rho$ has preference consistency across scenarios, we have that

$$
\begin{aligned}
& \Lambda\left(X_{\omega}\right)=\rho\left(X_{\omega} \mathbf{1}_{\Omega}^{\top}\right) \geq \Lambda\left(Y_{\omega}\right)=\rho\left(Y_{\omega} \mathbf{1}_{\Omega}^{\top}\right), \quad \forall \omega \in \Omega \quad \Rightarrow \quad \rho(X) \geq \rho(Y), \\
& \Lambda\left(X_{\omega}\right)=\rho\left(X_{\omega} \mathbf{1}_{\Omega}^{\top}\right) \leq \Lambda\left(Y_{\omega}\right)=\rho\left(Y_{\omega} \mathbf{1}_{\Omega}^{\top}\right), \quad \forall \omega \in \Omega \quad \Rightarrow \quad \rho(X) \leq \rho(Y) .
\end{aligned}
$$

Thus, we conclude that $\rho(X)=\rho(Y)$, and $\rho_{0}$ is well-defined. Clearly, $\rho_{0}$ is monotonic and positively homogeneous, from the monotonicity and positive homogeneity of $\Lambda$ and $\rho$. We show that $\rho_{0}$ is convex. For two vectors $x, y \in \mathcal{Q}^{\Omega}$, given a scalar $0 \leq \alpha \leq 1$, define $z=\alpha x+(1-\alpha) y$. Define vectors $\hat{X}, \hat{Y}, \hat{Z} \in \mathbb{R}^{|\Omega|}$ such that

$$
\rho_{0}(x)=\rho(\hat{X}), \quad \rho_{0}(y)=\rho(\hat{Y}), \quad \rho_{0}(z)=\rho(\hat{Z}) .
$$


Then, for all scenarios $\omega \in \Omega$,

$$
\Lambda\left(\hat{Z}_{\omega}\right)=z_{\omega}=\alpha x_{\omega}+(1-\alpha) y_{\omega}=\alpha \Lambda\left(\hat{X}_{\omega}\right)+(1-\alpha) \Lambda\left(\hat{Y}_{\omega}\right)
$$

From the risk convexity of $\rho$, we have that

$$
\rho_{0}(z)=\rho(\hat{Z}) \leq \alpha \rho(\hat{X})+(1-\alpha) \rho(\hat{Y})=\alpha \rho_{0}(x)+(1-\alpha) \rho_{0}(y) .
$$

This establishes the convexity of $\rho_{0}$. In addition,

$$
\rho_{0}\left(\mathbf{1}_{\Omega}\right)=\rho\left(a \mathbf{1}_{\Omega}^{\top}\right)
$$

where

$$
\Lambda(a)=\rho\left(a \mathbf{1}_{\Omega}^{\top}\right)=1
$$

It follows that $\rho_{0}\left(\mathbf{1}_{\Omega}\right)=1$. For part $(\mathrm{i}),-1 \in \mathcal{Q}$, we can show $\rho_{0}\left(-\mathbf{1}_{\Omega}\right)=-1$ similarly. Now, we can show that for part (i), for a scalar $\alpha \in \mathbb{R}$, by the sub-additivity (as a result of convexity and positive homogeneity) of $\rho_{0}$, we have that

$$
\rho_{0}\left(x+\alpha \mathbf{1}_{\Omega}\right) \geq \rho_{0}(x)+\alpha \rho_{0}\left(\mathbf{1}_{\Omega}\right)=\rho_{0}(x)+\alpha,
$$

and

$$
\rho_{0}\left(x-\alpha\left(-\mathbf{1}_{\Omega}\right)\right) \leq \rho_{0}(x)-\alpha \rho_{0}\left(-\mathbf{1}_{\Omega}\right)=\rho_{0}(x)+\alpha .
$$

Hence, $\rho_{0}$ has the cash invariance property $\rho_{0}\left(x+\alpha \mathbf{1}_{\Omega}\right)=\rho_{0}(x)+\alpha$ for part (i).

To summarize, for part (i) we have shown that $\rho_{0}$ is a coherent single-firm risk measure; for part (ii) we have shown that $\rho_{0}$ is a single-firm risk measure. From the definition of $\Lambda$ and $\rho_{0}$, we have the structural decomposition,

$$
\rho(X)=\left(\rho_{0} \circ \Lambda\right)(X)=\rho_{0}\left(\Lambda\left(X_{1}\right), \Lambda\left(X_{2}\right), \ldots, \Lambda\left(X_{|\Omega|}\right)\right) .
$$

For the converse of the theorem, suppose that $\Lambda$ is an aggregation function and $\rho_{0}$ is a base risk measure. Since $\Lambda$ and $\rho_{0}$ are monotonic, convex and positively homogeneous, it is clear that $\rho$ has the properties of monotonicity, convexity and positive homogeneity. The normalization condition is due to that of $\Lambda$ and the fact that $\rho_{0}\left(\mathbf{1}_{\Omega}\right)=1$. To show the preference consistency of $\rho$, consider two economies $X, Y \in \mathbb{R}^{|\mathcal{F}| \times|\Omega|}$ where, in every scenario $\omega \in \Omega$,

$$
\rho\left(X_{\omega} \mathbf{1}_{\Omega}^{\top}\right)=\left(\rho_{0} \circ \Lambda\right)\left(X_{\omega} \mathbf{1}_{\Omega}^{\top}\right) \geq \rho\left(Y_{\omega} \mathbf{1}_{\Omega}^{\top}\right)=\left(\rho_{0} \circ \Lambda\right)\left(Y_{\omega} \mathbf{1}_{\Omega}^{\top}\right)
$$

By the monotonicity of $\rho_{0}$, we have

$$
\Lambda\left(X_{\omega}\right) \geq \Lambda\left(Y_{\omega}\right), \quad \forall \omega \in \Omega
$$


Then, by using the monotonicity of $\rho_{0}$ again, we conclude that

$$
\rho(X)=\left(\rho_{0} \circ \Lambda\right)(X) \geq\left(\rho_{0} \circ \Lambda\right)(Y)=\rho(Y) .
$$

Now, we show the risk convexity of $\rho$. For any three economies $X, Y, Z \in \mathbb{R}^{|\mathcal{F}| \times|\Omega|}$ and any scalar $0 \leq \alpha \leq 1$, suppose for all scenarios $\omega \in \Omega$,

$\left(\rho_{0} \circ \Lambda\right)\left(Z_{\omega} \mathbf{1}_{\Omega}\right)=\rho\left(Z_{\omega} \mathbf{1}_{\Omega}\right)=\alpha \rho\left(X_{\omega} \mathbf{1}_{\Omega}\right)+(1-\alpha) \rho\left(Y_{\omega} \mathbf{1}_{\Omega}\right)=\alpha\left(\rho_{0} \circ \Lambda\right)\left(X_{\omega} \mathbf{1}_{\Omega}\right)+(1-\alpha)\left(\rho_{0} \circ \Lambda\right)\left(Y_{\omega} \mathbf{1}_{\Omega}\right)$.

We know that for part (i), we have that $\rho_{0}\left( \pm \mathbf{1}_{\Omega}\right)= \pm 1$, and for part (ii), we have $\rho_{0}\left(\mathbf{1}_{\Omega}\right)=1$. Thus, we can simplify the above equation, for both part (i)] and part (ii), as

$$
\Lambda\left(Z_{\omega}\right)=\alpha \Lambda\left(X_{\omega}\right)+(1-\alpha) \Lambda\left(Y_{\omega}\right), \quad \forall \omega \in \Omega
$$

Using the convexity of $\rho_{0}$, we conclude that, for all scenarios $\omega \in \Omega$,

$$
\rho(Z)=\left(\rho_{0} \circ \Lambda\right)(Z) \leq \alpha\left(\rho_{0} \circ \Lambda\right)(X)+(1-\alpha)\left(\rho_{0} \circ \Lambda\right)(Y)=\alpha \rho(X)+(1-\alpha) \rho(Y) .
$$

In addition, for part (i), there exists a vector $x \in \mathbb{R}^{|\mathcal{F}|}$ such that $\Lambda(x)<0$. So we have $\rho\left(x \mathbf{1}_{\Omega}^{\top}\right)=$ $\Lambda(x)<0$ and $\rho\left(\mathbf{1}_{\mathcal{E}}\right)=|\mathcal{F}|>0$. By positive homogeneity of $\rho$, we conclude that $\operatorname{Im} \rho=\mathbb{R}$. For part (ii) $\Lambda(x) \geq 0$, for all $x \in \mathbb{R}^{|\mathcal{F}|}$. So $\rho(X) \geq \rho_{0}\left(\mathbf{0}_{\mathcal{E}}\right)=0$, for all $X \in \mathbb{R}^{|\mathcal{F}| \times|\Omega|}$. We also know $\rho\left(\mathbf{1}_{\mathcal{E}}\right)=|\mathcal{F}|>0$. By positive homogeneity of $\rho$, we conclude that $\operatorname{Im} \rho=\mathbb{R}_{+}$.

\section{Variational Representations}

In this section, we develop two variational representations for systemic risk measures. In Section 3.1 we introduce a primal representation, where the systemic risk is the value of an optimization problem over a set of 'acceptable' economies. In Section 3.2, we develop a dual representation, where the systemic risk is the worst-case scaled expected loss across the economy. The dual representation provides a 'shadow price' to capture the systemic risk externality of the decision making of individual firms. This suggests a decentralized framework for systemic risk based decision-making that is further explored in the Online Supplement.

\subsection{Acceptance Sets and Primal Representation}

For the case of coherent single-firm risk measures, Artzner et al. (1999) describe a representation for the risk as the minimum quantity of cash that needs to be injected into each scenario such that the collection of outcomes is contained in a set of 'acceptable' outcomes. Motivated by this, we wish to construct a similar primal representation for systemic risk measures. In order to do this, we need the following definition. 
Definition 4 (Acceptance Set). Consider a finite set of entities $\mathcal{N}$. An acceptance set over $\mathcal{N}$ is a set $\mathcal{S} \subset \mathbb{R} \times \mathbb{R}^{|\mathcal{N}|}$, that is a non-empty closed convex cone, and that satisfies:

(i) Monotonicity: If $\left(m, x_{1}\right) \in \mathcal{S}, x_{2} \in \mathbb{R}^{|\mathcal{N}|}$, and $x_{1} \geq x_{2}$, then $\left(m, x_{2}\right) \in \mathcal{S}$.

(ii) Epigraph property: If $\left(m_{1}, x\right) \in \mathcal{S}, m_{2} \in \mathbb{R}$, and $m_{2} \geq m_{1}$, then $\left(m_{2}, x\right) \in \mathcal{S}$.

We take the set of entities $\mathcal{N}$ in Definition 4 either to be the collection of firms $\mathcal{F}$ or scenarios $\Omega$. In the former case, when $\mathcal{N}=\mathcal{F},(m, x) \in \mathbb{R} \times \mathbb{R}^{|\mathcal{F}|}$ is contained in the acceptance set if the crosssectional profile $x$ is considered acceptable at a given level of 'risk exposure' $m$. The monotonicity property suggests that, at a fixed level of risk exposure, loss profiles that are dominated by an acceptable profile are also acceptable. The epigraph property suggests that if a loss profile is acceptable at a certain level of risk exposure, it is also acceptable at higher levels of risk exposure. Similarly, when $\mathcal{N}=\Omega$, the acceptance set captures sets of risk exposures across scenarios, in addition to an overall risk measure, such that the per scenario risk exposures are acceptable relative to the overall risk measure. The properties of acceptance sets follow from the underlying properties of aggregation functions and base risk measures; in fact, we will shortly see that acceptance sets are epigraphs of these objects. Note that, relative to the case considered by Artzner et al. (1999), we require an additional dimension corresponding to the level of risk exposure of the regulator. If a cash invariance assumption held as in Artzner et al. (1999), this extra dimension could be eliminated, but in the present context it is necessary.

The following theorem provides a primal representation to a systemic risk measure, as the value of an optimization problem over a feasible set defined through acceptable sets:

Theorem 2 (Primal Representation). Suppose $\rho$ is a systemic risk measure. Then, there exist acceptance sets $\mathcal{A} \subset \mathbb{R} \times \mathbb{R}^{|\Omega|}$ and $\mathcal{B} \subset \mathbb{R} \times \mathbb{R}^{|\mathcal{F}|}$ over scenarios and firms, respectively, such that, for all economies $X \in \mathbb{R}^{|\mathcal{F}| \times|\Omega|}, \rho(X)$ can be expressed as the value of the optimization problem

$$
\begin{aligned}
\rho(X)=\underset{m, \ell}{\operatorname{minimize}} & m \\
\text { subject to } & (m, \ell) \in \mathcal{A}, \\
& \left(\ell_{\omega}, X_{\omega}\right) \in \mathcal{B}, \quad \forall \omega \in \Omega, \\
& m \in \mathbb{R}, \ell \in \mathbb{R}^{|\Omega|} .
\end{aligned}
$$

Further, if $\rho$ is characterized by a base risk measure $\rho_{0}$ and an aggregation function $\Lambda$, i.e., $\rho=$ $\rho_{0} \circ \Lambda$, then the acceptance sets can be taken as the epigraphs of $\rho_{0}$ and $\Lambda$, i.e.,

$$
\mathcal{A} \triangleq\left\{(m, z) \in \mathbb{R} \times \mathbb{R}^{|\Omega|}: m \geq \rho_{0}(z)\right\}, \quad \mathcal{B} \triangleq\left\{(\ell, x) \in \mathbb{R} \times \mathbb{R}^{|\mathcal{F}|}: \ell \geq \Lambda(x)\right\}
$$

Proof. Given $\rho$, by Theorem 1 a base risk measure $\rho_{0}$ and an aggregation function $\Lambda$ exist such that $\rho=\rho_{0} \circ \Lambda$. Define $\mathcal{A}$ and $\mathcal{B}$ to be their epigraphs through (11). From the properties of $\rho_{0}$ and $\Lambda$, it is clear that these are acceptance sets. 
Moreover, we have the epigraph representations

$$
\begin{aligned}
& \rho_{0}(\ell)=\underset{m \in \mathbb{R}}{\operatorname{minimize}} m \quad \Lambda(x)=\underset{\ell \in \mathbb{R}}{\operatorname{minimize}} \quad \ell \\
& \text { subject to }(m, \ell) \in \mathcal{A} . \quad \text { subject to }(\ell, x) \in \mathcal{B} \text {. }
\end{aligned}
$$

for all $\ell \in \mathbb{R}^{|\Omega|}, x \in \mathbb{R}^{|\mathcal{F}|}$. Using the fact that $\rho=\rho_{0} \circ \Lambda$, and the epigraph representation of $\rho_{0}$, we have for all $X \in \mathbb{R}^{|\mathcal{F}| \times|\Omega|}$,

$$
\begin{aligned}
& \rho(X)=\underset{m \in \mathbb{R}}{\operatorname{minimize}} m \\
& \text { subject to }\left(m, \Lambda\left(X_{1}\right), \ldots, \Lambda\left(X_{|\Omega|}\right)\right) \in \mathcal{A} \text {. }
\end{aligned}
$$

Using the fact that $\mathcal{A}$ is monotonic, and applying the epigraph representation of $\Lambda$, the result follows.

The primal program (10) is easily interpreted: the vector of decision variables $\ell$ defines the regulator's minimal risk exposure in each scenario given the corresponding cross-sectional loss profile, while the scalar decision variable $m$ is overall systemic risk given the vector $\ell$ of risk exposures across scenarios.

\subsection{Dual Representation}

In this section, we define a dual representation for systemic risk measures. This variational representation provides an alternative way to compute systemic risk measures and an alternative interpretation of their meaning. Moreover, it provides certain computational and operational advantages. In Section 4, we show that the dual representation also provides the basis of a risk attribution rule.

To begin, suppose $\rho=\rho_{0} \circ \Lambda$ is a systemic risk measure. As in Theorem 2, take the epigraphs of $\rho_{0}$ and $\Lambda$ as the acceptance sets $\mathcal{A}$ and $\mathcal{B}$, respectively. Define

$$
\begin{aligned}
& \mathcal{A}^{*} \triangleq\left\{\left(\pi_{0}, \hat{\pi}\right) \in \mathbb{R} \times \mathbb{R}^{|\Omega|}: \pi_{0} m-\hat{\pi}^{\top} \ell \geq 0, \forall(m, \ell) \in \mathcal{A}\right\}, \\
& \mathcal{B}^{*} \triangleq\left\{\left(\xi_{0}, \hat{\xi}\right) \in \mathbb{R} \times \mathbb{R}^{|\mathcal{F}|}: \xi_{0} \ell-\hat{\xi}^{\top} x \geq 0, \forall(\ell, x) \in \mathcal{B}\right\} .
\end{aligned}
$$

Up to a sign change, $\mathcal{A}^{*}$ and $\mathcal{B}^{*}$ are the dual cones to $\mathcal{A}$ and $\mathcal{B}$, respectively. Then, the following theorem, whose full proof can be found in the Online Supplement, holds.

Theorem 3. Suppose $\rho=\rho_{0} \circ \Lambda$ is a systemic risk measure characterized by an aggregation function $\Lambda$ and a base risk measure $\rho_{0}$. Then, for all economies $X \in \mathbb{R}^{|\mathcal{F}| \times|\Omega|}, \rho(X)$ can be expressed as the 
value of the optimization problem

$$
\begin{array}{lll}
\rho(X)=\underset{\bar{\pi}, \Xi}{\operatorname{maximize}} & \sum_{i \in \mathcal{F}} \sum_{\omega \in \Omega} \Xi_{i, \omega} X_{i, \omega} & \\
\text { subject to } & (1, \bar{\pi}) \in \mathcal{A}^{*}, & \\
& \left(\bar{\pi}_{\omega}, \Xi_{\omega}\right) \in \mathcal{B}^{*}, \quad \forall \omega \in \Omega, \\
& \bar{\pi} \in \mathbb{R}^{|\Omega|}, \Xi \in \mathbb{R}^{|\mathcal{F}| \times|\Omega|} . &
\end{array}
$$

In addition, feasible points $(\bar{\pi}, \Xi)$ for this problem must satisfy

$$
\bar{\pi} \geq \mathbf{0}_{\Omega}, \quad \mathbf{1}_{\Omega}^{\top} \bar{\pi} \leq 1, \quad \Xi \geq \mathbf{0}_{\mathcal{E}}, \quad \mathbf{1}_{\mathcal{F}}^{\top} \Xi \leq|\mathcal{F}| \bar{\pi}^{\top}
$$

In order to interpret the dual problem (14), observe that (15) implies that, for feasible $(\bar{\pi}, \Xi), \bar{\pi}$ is a sub-stochastic vector. This can be interpreted as a probability distribution over the augmented set of scenarios $\Omega \cup\left\{\omega_{0}\right\}$, where $\omega_{0}$ is an additional, artificial scenario in which every firm has a 0 outcome. Define the matrix $\hat{\Xi} \in \mathbb{R}^{|\mathcal{F}| \times|\Omega|}$ by

$$
\hat{\Xi}_{i, \omega} \triangleq \begin{cases}\Xi_{i, \omega} / \pi_{\omega} & \text { if } \pi_{\omega}>0 \\ 0 & \text { otherwise }\end{cases}
$$

for each firm $i$ and scenario $\omega$. Then, the objective in (14) becomes

$$
\sum_{\omega \in \Omega} \bar{\pi}_{\omega} \sum_{i \in \mathcal{F}} \hat{\Xi}_{i, \omega} X_{i, \omega}
$$

where (15) implies that

$$
\hat{\Xi} \geq \mathbf{0}_{\mathcal{E}}, \quad \sum_{i \in \mathcal{F}} \hat{\Xi}_{i, \omega} \leq|\mathcal{F}|, \forall \omega \in \Omega
$$

In other words, the dual objective is the worst-case expected loss, over some set of feasible probability distributions $\hat{\pi}$ and scaling functions $\hat{\Xi}$, of a scaled economy in which the participation of the $|\mathcal{F}|$ firms in the economy in scenario $\omega$ is rescaled according to the vector $\hat{\Xi}_{1, \omega}, \ldots, \hat{\Xi}_{|\mathcal{F}| \omega}$. This is analogous to the robust interpretation of a single-firm coherent risk measure as a worst-case expected loss.

The following is an immediate corollary of Theorem 3 .

Corollary 1. Suppose that $\rho$ is a systemic risk measure with dual representation (14). Given an economy $X$, if $\left(\bar{\pi}^{*}, \Xi^{*}\right)$ is a dual optimal solution, then $\Xi^{*}$ is a subgradient of $\rho$ at $X$.

Corollary 1 suggests another interpretation of the optimal dual solution $\Xi^{*}$ for an economy $X$. The quantity $\Xi_{i, \omega}^{*}$ is the minimal marginal increase in systemic risk as function of a marginal increase in the losses of firm $i$ in scenario $\omega$. In other words, $\Xi_{i, \omega}^{*}$ captures the externalities imposed by the decision-making of a firm on the system regulator, and hence is a shadow price for systemic risk. Note that these shadow prices can vary both by scenario - incremental losses in some scenarios 
may have a much larger impact than in other scenarios - and by the identity of the firm. These shadow prices could be used to coordinate decision-making by individual firms with the goals of the regulator. For example, it is possible to design tax schemes based on these prices, in the spirit of Acharya et al. (2010a), such that individual firms optimize their portfolios to distribute profits and losses across scenarios in a way that is aligned with the concerns of the regulator. This topic is further explored in the Online Supplement.

The dual optimization problem (14) may also lead to useful decentralized schemes for computing systemic risk. Here, a centralized regulator can seek to choose optimal values for the dual variables $(\bar{\pi}, \Xi)$, while relying on constituent firms to compute their individual weighted profits and losses, scaled according to each putative choice of dual variables. The utility of the dual representation from analytical, operational, and computational perspectives is illustrated by the following examples.

Example 8 (Total P\&L). Consider the total profit and loss aggregation function $\Lambda_{\text {total }}$, defined by (1). For this aggregation function, it is easy to see that

$$
\mathcal{B}^{*}=\left\{\left(\xi_{0}, \xi_{0} \mathbf{1}_{\mathcal{F}}\right) \in \mathbb{R} \times \mathbb{R}^{|\mathcal{F}|}: \xi_{0} \in \mathbb{R}_{+}\right\}
$$

Then, the dual representation (14) takes the simplified form

$$
\begin{aligned}
\rho(X)=\operatorname{\pi n}_{\bar{\pi} \in \mathbb{R}^{|\Omega|}}^{\operatorname{maximize}} & \sum_{i \in \mathcal{F}} \sum_{\omega \in \Omega} \bar{\pi}_{\omega} X_{i, \omega} \\
& \text { subject to } \quad(1, \bar{\pi}) \in \mathcal{A}^{*} .
\end{aligned}
$$

In Example 8, the base risk measure (and thus the constraint set $\mathcal{A}^{*}$ ) has not been specified. However, independent of this choice, given an optimal dual solution $\bar{\pi}^{*}$, a shadow price for systemic risk for each firm $i$ in a scenario $\omega$ is given by $\bar{\pi}_{\omega}^{*}$ and is independent of the identity of the firm. This is consistent with the choice of aggregation function: the impact of a marginal increase in the loss of any firm is the same, since the sum total of all profits and losses is of concern.

Example 9 (Total Loss). Consider the total loss aggregation function $\Lambda_{\text {loss }}$, defined by (3). Then, we have that

$$
\mathcal{B}^{*}=\left\{\left(\xi_{0}, \hat{\xi}\right) \in \mathbb{R} \times \mathbb{R}^{|\mathcal{F}|}: \mathbf{0}_{\mathcal{F}} \leq \hat{\xi} \leq \xi_{0} \mathbf{1}_{\mathcal{F}}\right\} .
$$

Thus, the dual representation (14) takes the simplified form

$$
\begin{aligned}
& \rho(X)=\underset{\bar{\pi} \in \mathbb{R}^{|\Omega|}}{\operatorname{maximize}} \sum_{i \in \mathcal{F}} \sum_{\omega \in \Omega} \bar{\pi}_{\omega}\left(X_{i, \omega}\right)^{+} \\
& \text {subject to }(1, \bar{\pi}) \in \mathcal{A}^{*} \text {. }
\end{aligned}
$$

In Example 9, given an optimal dual solution $\bar{\pi}^{*}$, a shadow price for systemic risk for firm $i$ in scenario $\omega$ is given by $\bar{\pi}_{\omega}^{*}$ if $X_{i, \omega} \geq 0$, and is 0 otherwise. This is because a firm can only marginally impact the systemic risk in scenarios where it is not profitable. 
Example 10 (CVaR). Suppose, given $0<\zeta<1$, the aggregation function is taken to be

$$
\Lambda_{\mathrm{CVaR}}(x) \triangleq \inf _{\ell \in \mathbb{R}}|\mathcal{F}| \ell+\frac{1}{\zeta} \sum_{i \in \mathcal{F}}\left(x_{i}-\ell\right)^{+}
$$

This corresponds to the aggregate total profits and losses of the worst $\zeta$-percentile of firms in the cross-sectional profile, i.e., it is analogous to the $\mathrm{CVaR}_{\zeta}$ risk measure of (2), but taken across firms rather than scenarios. Then, we have that

$$
\mathcal{B}^{*}=\left\{\left(\xi_{0}, \hat{\xi}\right) \in \mathbb{R} \times \mathbb{R}^{|\mathcal{F}|}: \mathbf{0}_{\mathcal{F}} \leq \hat{\xi} \leq \frac{\xi_{0}}{\zeta} \mathbf{1}_{\mathcal{F}}, \mathbf{1}_{\mathcal{F}}^{\top} \hat{\xi}=\xi_{0}|\mathcal{F}| .\right\}
$$

Thus, the dual representation (14) takes the simplified form

$$
\begin{aligned}
\rho(X)=\underset{\bar{\pi}, \Xi}{\operatorname{maximize}} & \sum_{i \in \mathcal{F}} \sum_{\omega \in \Omega} \Xi_{i, \omega} X_{i, \omega} \\
\text { subject to } & (1, \bar{\pi}) \in \mathcal{A}^{*}, \\
& \mathbf{0}_{\mathcal{E}} \leq \Xi \leq \frac{1}{\zeta} \bar{\pi} \mathbf{1}_{\Omega}^{\top}, \\
& \mathbf{1}_{\mathcal{F}}^{\top} \Xi=|\mathcal{F}| \bar{\pi}, \\
& \bar{\pi} \in \mathbb{R}^{|\Omega|}, \Xi \in \mathbb{R}^{|\mathcal{F}| \times|\Omega|} .
\end{aligned}
$$

In Example 10, a shadow price for systemic risk $\Xi_{i, \omega}^{*}$, in general, depends both on the identity of the firm $i$ and the particular scenario $\omega$.

\section{Risk Attribution}

In this section, we consider the problem of attributing or allocating the systemic risk across the firms that compose the economy. The spirit here is to identify systemically risky institutions, and quantify their overall impact on the risk in the economy. Motivated by the discussion of shadow prices in Section 3.2, consider the following definition:

Definition 5 (Risk Attribution). Suppose $\rho$ is a systemic risk measure, with dual decomposition (14). For each economy $X \in \mathbb{R}^{|\mathcal{F}| \times|\Omega|}$, define $\mathcal{M}(X) \subset \mathbb{R}^{|\mathcal{F}| \times|\Omega|}$ to be the set of dual optimal solutions for $\rho(X)$. Given a shadow price for systemic risk $\Xi^{*} \in \mathcal{M}(X)$, we define a vector $y^{*}\left(X, \Xi^{*}\right) \in \mathbb{R}^{|\mathcal{F}|}$, with component

$$
y_{i}^{*}\left(X, \Xi^{*}\right) \triangleq \sum_{\omega \in \Omega} \Xi_{i, \omega}^{*} X_{i, \omega}
$$

as the systemic risk attributable to firm $i$. We define set of all attribution vectors

$$
\mathcal{Y} \triangleq\left\{y^{*}\left(X, \Xi^{*}\right): \Xi^{*} \in \mathcal{M}(X)\right\}
$$

Note that the attribution rule is unique if the dual optimal solution for $\rho(X)$ is unique at $X$.

This definition allocates systemic risk to each firm according its entire profile of profits and losses 
across scenarios, where each profit or loss is valued according to the appropriate shadow price for systemic risk. Note that the risk allocation is an immediate by-product of the dual representation, and hence requires no computation if the dual solution is available.

The allocation of Definition 5 has a number of desirable properties. First, since Theorem 3 guarantees that the dual optimum equals $\rho(X)$, it is immediate that

$$
\rho(X)=\sum_{i \in \mathcal{F}} y_{i}^{*}, \quad \forall y^{*} \in \mathcal{Y}
$$

In order words, the individual risk attributions add up to the total systemic risk. Second, following Corollary 1. the sensitivity of the attribution $y_{i}$ of firm $i$ to a change in the loss $X_{i, \omega}$ in some scenario $\omega$ is precisely the shadow price for systemic risk. Hence, the local incentives created by this allocation are aligned with the systemic risk objective. Finally, the risk attribution that we propose has the following fairness property:

Theorem 4. Fix a systemic risk measure $\rho$. Let $X \in \mathbb{R}^{|\mathcal{F}| \times|\Omega|}$ denote a given economy.

For a vector $\alpha \in \mathbb{R}_{+}^{\mathcal{F}}$, define $r(\alpha)$ to be the systemic risk associated with an economy $\alpha * X$ that has outcomes for firm $i$ in scenario $\omega$ given by $\alpha_{i} X_{i, \omega}$. Then, for any risk attribution $y^{*} \in \mathcal{Y}$, $\alpha^{\top} y^{*} \leq r(\alpha)$.

Proof. From the dual representation in Theorem 3 , we have that

$$
\begin{aligned}
& r(\alpha)=\operatorname{maximize}_{\bar{\pi}, \Xi} \sum_{i \in \mathcal{F}} \alpha_{i} \sum_{\omega \in \Omega} \Xi_{i, \omega} X_{i, \omega} \\
& \text { subject to }(1, \bar{\pi}) \in \mathcal{A}^{*} \text {, } \\
& \left(\bar{\pi}_{\omega}, \Xi_{\omega}\right) \in \mathcal{B}^{*}, \quad \forall \omega \in \Omega, \\
& \bar{\pi} \in \mathbb{R}^{|\Omega|}, \Xi \in \mathbb{R}^{|\mathcal{F}| \times|\Omega|} \text {. }
\end{aligned}
$$

Suppose $y^{*}$ is obtained by a dual optimal solution $\Xi^{*}$. Since $\Xi^{*}$ is a feasible solution of the dual representation of $\rho(X)$ in (14), $\Xi^{*}$ is also a feasible solution of the dual representation of $r(\alpha)$ in 16), for any $\alpha$. The objective value achieved by $\Xi^{*}$ in 16 is $\alpha^{\top} y^{*}$, which can be no greater than the optimal value $r(\alpha)$, i.e., $\alpha^{\top} y^{*} \leq r(\alpha)$.

Theorem 4 is a 'no-undercut' result, in the spirit of Denault (2001). Here, $r(\alpha)$ is the systemic risk associated with an economy where each firm $i$ participates proportionally to the factor $\alpha_{i} \geq 0$. The result states that, if a fractional coalition of firms specified by the vector $\alpha$ form a new economy $\alpha * X$, the systemic risk of that economy $r(\alpha) \triangleq \rho(\alpha * X)$ is at least as large as the weighted sum of risk attributed to the firms in the original economy. Thus, the risk attribution is fair: the risk attributed to any fractional coalition is no greater than it would incur as a standalone economy.

The risk attribution we propose is closely related to prices of Aumann and Shapley (1974) for allocating the cost in a fractional coalition game. If we assume that $r$ is differentiable at the point $\alpha=\mathbf{1}_{\mathcal{F}}$, then by positive homogeneity, it is differentiable on the ray $\left\{t \mathbf{1}_{\mathcal{F}}: t \geq 0\right\}$. The 
Aumann-Shapley prices are then defined by

$$
y^{\mathrm{AS}} \triangleq \int_{0}^{1} \nabla r\left(t \mathbf{1}_{\mathcal{F}}\right) d t=\nabla r\left(\mathbf{1}_{\mathcal{F}}\right)
$$

The last equality follows from the fact that $r$ is positively homogeneous, and is sometimes referred to as the Euler allocation rule or gradient allocation rule (Denault, 2001, Buch and Dorfleitner, 2008). In fact, when these latter attribution rules are well-defined, they correspond with our notion of risk allocation:

Theorem 5. Given a systemic risk measure $\rho$ and an economy $X \in \mathbb{R}^{|\mathcal{F}| \times|\Omega|}$, if $\rho$ is differentiable at $X$, then the risk attribution $y^{*}$ is unique and coincides with the Aumann-Shapley prices $y^{A S}$.

Proof. Under the hypothesis, $r(\alpha)$ defined in Theorem 4 is differentiable at $\alpha=\mathbf{1}_{\mathcal{F}}$. Observing that the constraint set in (16) is compact, we can apply Danskin's theorem (Bertsekas, 1999) to (16) to obtain

$$
\frac{\partial r(\alpha)}{\partial \alpha_{i}}=\sum_{\omega \in \Omega} \Xi_{i, \omega}^{*} X_{i, \omega}
$$

for all firms $i$, where $\Xi^{*} \in \mathbb{R}^{|\mathcal{F}| \times|\Omega|}$ is the unique dual optimal solution for $\rho(X)$. Therefore, $y^{A S}=y^{*}$.

Note that the work of Denault (2001), Fischer (2003), and Buch and Dorfleitner (2008) suggests the gradient allocation rule for risk attribution in a portfolio setting; that setting is a special case of systemic risk measure corresponding to the total P\&L aggregation function $\Lambda_{\text {total }}$ of (1). In that case, the gradient allocation rule is identical to our dual risk attribution $y^{*}$. Our dual risk attribution $y^{*}$, however, requires no differentiability assumption, and can apply to a more general class of aggregation functions. When $\rho$ is not differentiable at $X$, several attribution rules $y^{*}$ are possible and how to choose among them may require further investigation. Related discussion on risk attribution can be found in the work of Tsanakas (2009) and Cherny and Orlov (2011).

\section{Homogeneous Systemic Risk Measures}

In this section, we extend our analysis to value-at-risk-like measures that are monotonic, positively homogeneous but non-convex. Value-at-risk (VaR) (see, e.g., Jorion, 2006) is a single-firm risk measure of particular importance, because it is extensively used in the practice of financial risk management. Originally developed by practitioners in the financial industry, it is widely employed both by firm managers and regulators to compute and manage market risk - in fact, VaR is the preferred measurement of market risk of the Basel II regulatory regime. The popularity of VaR as a single-firm risk measure has motivated a number of VaR-based measures of systemic risk, such as the CoVaR measure proposed by Adrian and Brunnermeier (2009).

The VaR at a confidence level $\zeta \in(0,1)$ is defined as follows: suppose $x \in \mathbb{R}^{|\Omega|}$ is a vector of losses across scenarios $\Omega$, and that $p \in \mathbb{R}_{+}^{|\Omega|}$ with $\mathbf{1}_{\Omega}^{\top} p=1$ is a probability distribution over $\Omega$. 
Then, the VaR of the random loss $x$ is the minimum loss threshold value $\ell$ such that the probability of the loss exceeding $\ell$ is at most $1-\zeta$, i.e.,

$$
\operatorname{VaR}_{\zeta}(x) \triangleq \inf \left\{\ell \in \mathbb{R}: \sum_{\omega \in \Omega: x_{\omega}>\ell} p_{\omega} \leq 1-\zeta\right\} .
$$

From the definition of $\mathrm{VaR}$, it is clear that this risk measure is positively homogeneous, monotonic, normalized, and cash invariant, i.e., it satisfies conditions (i), (ii), (iv), and (v) of Definition 1. However, it is not convex.

The lack of convexity is the principal difference between homogeneous risk measures and the (convex) risk measures defined in Section 2, Aside from their wide use in practice, homogeneous risk measures have also generated some interest in the literature (e.g., Kou et al., 2009, CerreiaVioglio et al., 2010). Our goal here is to illustrate the impact of the absence of this axiom on our framework for systemic risk.

In what follows, we investigate the impact of dropping the convexity requirement for systemic risk measures. In Section 5.1, we give a complete structural decomposition for homogeneous risk measures. The benefit from this analysis is two-fold. First, as in the case with convex systemic risk measures discussed in the previous sections, the characterization gives us a rule for constructing homogeneous systemic risk measures from a homogeneous base risk measure and homogeneous aggregation functions. The second and equally important benefit is that the characterization elucidates the implicit assumptions that are being made when one combines single-firm homogeneous risk measures to create a systemic risk measure. As a by-product of our characterization, in Section 5.2 we show that a homogeneous systemic measures have a convex representation. Finally, in Section 5.4 we consider some examples of homogeneous systemic risk measures.

\subsection{Structural Decomposition}

Motivated by the discussion above, we define homogeneous systemic risk measures as follows:

Definition 6 (Homogeneous Systemic Risk Measure). A homogeneous systemic risk measure is a function $\rho: \mathbb{R}^{|\mathcal{F}| \times|\Omega|} \rightarrow \mathbb{R}$ that satisfies the following conditions, for all economies $X, Y \in \mathbb{R}^{|\mathcal{F}| \times|\Omega|}$ :

(I) Monotonicity: $X \geq Y$ implies $\rho(X) \geq \rho(Y)$.

(II) Positive homogeneity: For all $\alpha \geq 0$ and $\rho(\alpha X)=\alpha \rho(X)$.

(III) Preference consistency: $\rho\left(X_{\omega} \mathbf{1}_{\Omega}^{\top}\right) \geq \rho\left(Y_{\omega} \mathbf{1}_{\Omega}^{\top}\right), \forall \omega \in \Omega$, implies $\rho(X) \geq \rho(Y)$.

$(I V)$ Normalization: $\rho\left(\mathbf{1}_{\mathcal{E}}\right)=|\mathcal{F}|$.

We define homogeneous single-firm risk measures and homogeneous aggregation functions as follows: 
Definition 7 (Homogeneous Single-Firm Risk Measure). A homogeneous single-firm risk measure is a function ${ }^{9} \rho: \mathbb{R}^{|\Omega|} \rightarrow \mathbb{R}$ that, for all loss vectors $x, y \in \mathbb{R}^{|\Omega|}$ of a single firm, satisfies the following conditions:

(i) Monotonicity: If $x \geq y$, then $\rho(x) \geq \rho(y)$.

(ii) Positive homogeneity: For all non-negative scalars $\alpha \geq 0, \rho(\alpha x)=\alpha \rho(x)$.

(iii) Normalization: $\rho\left(\mathbf{1}_{\Omega}\right)=1$.

Definition 8 (Homogeneous Aggregation Function). A function $\Lambda: \mathbb{R}^{|\mathcal{F}|} \rightarrow \mathbb{R}$ over cross-sectional loss profiles of firms is a homogeneous aggregation function if, for all cross-sectional loss profiles $x, y \in \mathbb{R}^{|\mathcal{F}|}$, it satisfies:

(i) Monotonicity: If $x \geq y$, then $\Lambda(x) \geq \Lambda(y)$.

(ii) Positive homogeneity: For all $\alpha \geq 0, \Lambda(\alpha x)=\alpha \Lambda(x)$.

(iii) Normalization: $\Lambda\left(\mathbf{1}_{\mathcal{F}}\right)=|\mathcal{F}|$.

The (convex) single-firm risk measures and the (convex) aggregation functions defined earlier in Definitions 1 and 3 are in fact also homogeneous single measures and aggregation functions, respectively; in addition, those functions are also convex.

homogeneous systemic risk measures admit a structural decomposition analogous to that of Theorem 1, as follows:

Theorem 6. (i) A function $\rho: \mathbb{R}^{|\mathcal{F}| \times|\Omega|} \rightarrow \mathbb{R}$ is a homogeneous systemic risk measure with image $\operatorname{Im} \rho=\mathbb{R}$ if and only if there exist a homogeneous aggregation function $\Lambda: \mathbb{R}^{|\mathcal{F}|} \rightarrow \mathbb{R}$ and a homogeneous single-firm risk measure $\rho_{0}: \mathbb{R}^{|\Omega|} \rightarrow \mathbb{R}$ with $\rho_{0}\left( \pm \mathbf{1}_{\Omega}\right)= \pm 1$ such that $\rho$ is the composition of $\rho_{0}$ and $\Lambda$, i.e.,

$$
\rho(X)=\left(\rho_{0} \circ \Lambda\right)(X) \triangleq \rho_{0}\left(\Lambda\left(X_{1}\right), \Lambda\left(X_{2}\right), \ldots, \Lambda\left(X_{|\Omega|}\right)\right)
$$

(ii) A function $\rho: \mathbb{R}^{|\mathcal{F}| \times|\Omega|} \rightarrow \mathbb{R}$ is a homogeneous systemic risk measure with image $\operatorname{Im} \rho=\mathbb{R}_{+}$ if and only if there exist a homogeneous aggregation function $\Lambda: \mathbb{R}^{|\mathcal{F}|} \rightarrow \mathbb{R}$ with $\operatorname{Im} \Lambda=\mathbb{R}_{+}$ and a homogeneous single-firm risk measure $\rho_{0}: \mathbb{R}_{+}^{|\Omega|} \rightarrow \mathbb{R}$ such that $\rho$ is the composition of $\rho_{0}$ and $\Lambda$, i.e.,

$$
\rho(X)=\left(\rho_{0} \circ \Lambda\right)(X) \triangleq \rho_{0}\left(\Lambda\left(X_{1}\right), \Lambda\left(X_{2}\right), \ldots, \Lambda\left(X_{|\Omega|}\right)\right)
$$

Proof. The proof is a simplified version of the proof of Theorem 1. since, in this case, it is not necessary to establish convexity. It is thus omitted.

\footnotetext{
${ }^{9}$ As was the case with Definition 1 , we sometimes consider homogeneous single-firm risk measures $\rho: \mathbb{R}_{+}^{|\Omega|} \rightarrow \mathbb{R}$ defined only on the positive orthant. In that case, we assume that conditions (i) (ii) are satisfied for all $x, y \in \mathbb{R}_{+}^{|\Omega|}$.
} 
Observe that, comparing Theorem 1 and Theorem 6 , preference consistency condition (III) is key to establishing this structural decomposition. The other conditions, namely homogeneity and monotonicity, imply these same properties for the single-firm risk measure $\rho_{0}$ and the aggregation function $\Lambda$.

\subsection{Convex Representation}

In this section, we develop a convex representation for homogeneous (non-convex) systemic risk measures as the pointwise minima of a collection of convex risk functions. To begin, consider the following lemma, a proof of which that follows the argument of Castellani (2000) is provided in the Online Supplement:

Lemma 1. A function $g: \mathbb{R}^{n} \rightarrow \mathbb{R}$ is positively homogeneous and monotonic if and only if there exists an index set $\mathcal{S}$ where, for each $s \in \mathcal{S}, g^{(s)}: \mathbb{R}^{n} \rightarrow \mathbb{R} \cup\{\infty\}$ is a positively homogeneous, monotonic, and convex extended real-valued function, such that

$$
g(x)=\underset{s \in \mathcal{S}}{\operatorname{minimize}} g^{(s)}(x), \quad \forall x \in \mathbb{R}^{n} .
$$

The following is a corollary of Lemma 1. It establishes a representation for homogeneous systemic risk measures in terms of convex single-firm risk measures and aggregation functions.

Corollary 2. Suppose $\rho$ is a homogeneous systemic risk measure with $\operatorname{Im} \rho=\mathbb{R}$. For all economies $X \in \mathbb{R}^{|\mathcal{F}| \times|\Omega|}, \rho(X)$ can be expressed as the value of the optimization problem

$$
\rho(X)=\operatorname{minimize}_{t \in \mathcal{T}, s_{1} \in \mathcal{S}, \ldots, s_{|\Omega|} \in \mathcal{S}} \rho^{(t)}\left(\Lambda^{\left(s_{1}\right)}\left(X_{1}\right), \ldots, \Lambda^{\left(s_{|\Omega|}\right)}\left(X_{|\Omega|}\right)\right)
$$

Here, $\mathcal{S}$ and $\mathcal{T}$ are index sets. For each $s \in \mathcal{S}$, the aggregation function $\Lambda^{(s)}: \mathbb{R}^{|\mathcal{F}|} \rightarrow \mathbb{R} \cup\{\infty\}$ satisfies conditions (i) (iii) of Definition 3 (i.e., monotonicity, positive homogeneity, convexity). For each $t \in \mathcal{T}$, the single-firm risk measure $\rho_{0}^{(t)}: \mathbb{R}^{|\Omega|} \rightarrow \mathbb{R} \cup\{\infty\}$ satisfies conditions (i) (iii) of Definition 1 (i.e., monotonicity, positive homogeneity, convexity).

Proof. The result follows by first applying Theorem 6 to obtain the representation $\rho=\rho_{0} \circ \Lambda$ in terms of a homogeneous single-firm base risk measure $\rho_{0}$ and a homogeneous aggregation function $\Lambda$, and then applying Lemma 1 to $\rho_{0}$ and $\Lambda$.

As an example of this construction, consider the following:

Example $11(\mathrm{VaR})$. Define the single-firm base measure $\rho_{0}(x)=\operatorname{VaR}_{\zeta}(x)$ to be the value-at-risk defined in (17), given a uniform probability distribution $p \triangleq \mathbf{1}_{\Omega} /|\Omega|$ and a confidence level $\zeta \in(0,1)$. In this case, we have that $\rho_{0}(x)=x_{\left[k^{*}\right]}$, where $k^{*} \triangleq\lceil\zeta|\Omega|\rceil$ and, for each $1 \leq k \leq|\Omega|, x_{[k]}$ is the kth order statistic of the vector $x$. Define $q^{(k)} \in \mathbb{R}^{|\Omega|}$ to be a vector with the first $|\Omega|-k$ components equal to 1 , and the rest equal to 0 , and define $\mathcal{T} \subset \mathbb{R}^{|\Omega| \times|\Omega|}$ to be the set of $|\Omega| \times|\Omega|$ permutation 
matrices. Then, we have that

$$
\rho_{0}(x)=\sum_{k=k^{*}}^{|\Omega|} x_{[k]}-\sum_{k=k^{*}+1}^{|\Omega|} x_{[k]}=\max _{P \in \mathcal{T}} x^{\top} P q^{\left(k^{*}\right)}-\max _{P \in \mathcal{T}} x^{\top} P q^{\left(k^{*}+1\right)}=\min _{P \in \mathcal{T}} \rho^{(P)}(x),
$$

where, for each $P \in \mathcal{T}$,

$$
\rho^{(P)}(x) \triangleq \max _{Q \in \mathcal{T}} x^{\top}\left(Q q^{\left(k^{*}\right)}-P q^{\left(k^{*}+1\right)}\right)
$$

is a convex base risk measure.

\subsection{Risk Attribution}

Next, we discuss the issue of attributing the total risk $\rho(X)$ across the $|\mathcal{F}|$ firms in the economy. We show below that a good risk attribution rule exists for a subset of homogeneous systemic risk measures that includes VaR.

For a systemic risk measure $\rho$, an economy $X$, and a vector $\alpha \in \mathbb{R}_{+}^{|\mathcal{F}|}$, define $r_{\rho}(\alpha) \triangleq \rho(\alpha * X)$, where $\alpha * X$ is defined by setting the outcomes for firm $i$ in scenario $\omega$ to $\alpha_{i} X_{i, \omega}$. In this setting, a risk allocation function $\Psi^{|\mathcal{F}|}$ takes as inputs two arguments, namely the function $r_{\rho}: \mathbb{R}_{+}^{|\mathcal{F}|} \rightarrow \mathbb{R}$ and a vector of fractional participation $\alpha \in \mathbb{R}_{+}^{|\mathcal{F}|}$, and returns a risk allocation to each firm. The following are certain desirable properties for $\Psi$ that are typically assumed in the literature (e.g., Billera and Heath, 1982). Suppose we have any two systemic risk measures $\rho_{1}$ and $\rho_{2}$.

1. Full risk allocation: $\alpha^{\top} \Psi^{|\mathcal{F}|}\left(r_{\rho}, \alpha\right)=r_{\rho}(\alpha)$.

2. Additivity: $\Psi^{|\mathcal{F}|}\left(r_{\rho_{1}}, \alpha\right)+\Psi^{|\mathcal{F}|}\left(r_{\rho_{2}}, \alpha\right)=\Psi^{|\mathcal{F}|}\left(r_{\rho_{1}}+r_{\rho_{2}}, \alpha\right)$.

3. Monotonicity: If $r_{\rho_{1}}(\alpha) \geq r_{\rho_{2}}(\alpha)$, for all $\alpha \in \mathbb{R}_{+}^{|\mathcal{F}|}$, then

$$
\Psi^{|\mathcal{F}|}\left(r_{\rho_{1}}, \alpha\right) \geq \Psi^{|\mathcal{F}|}\left(r_{\rho_{2}}, \alpha\right), \quad \forall \alpha \in \mathbb{R}_{+}^{|\mathcal{F}|} .
$$

4. Rescaling invariance: For a vector $\beta \in \mathbb{R}_{+}^{|\mathcal{F}|}$, define $\beta * \alpha \triangleq\left(\beta_{1} \alpha_{1}, \beta_{2} \alpha_{2}, \ldots, \beta_{|\mathcal{F}|} \alpha_{|\mathcal{F}|}\right)$. If $r_{\rho_{1}}(\alpha)=r_{\rho_{2}}(\beta * \alpha)$, then

$$
\Psi_{i}^{|\mathcal{F}|}\left(r_{\rho_{1}}, \beta^{-1} * \alpha\right)=\beta_{i} \Psi_{i}^{|\mathcal{F}|}\left(r_{\rho_{2}}, \alpha\right), \quad \forall i \in \mathcal{F}
$$

5. Consistency: If there is a function $\bar{r}$ such that $r(\alpha)=\bar{r}\left(\mathbf{1}_{\mathcal{F}}^{\top} \alpha\right)$, then

$$
\Psi_{i}^{|\mathcal{F}|}(r, \alpha)=\Psi^{1}\left(\bar{r}, \mathbf{1}_{\mathcal{F}}^{\top} \alpha\right), \quad \forall i \in \mathcal{F}
$$

Here, $\Psi^{1}$ is a single-firm cost allocation function.

Note that for the general class of differentiable risk functions $r$, Billera and Heath (1982) show that Aumann-Shapley prices are the only attribution rule that satisfies all five properties. For 
piecewise linear risk functions, Haimanko (2001) shows that the Mertens (1988) mechanism is the unique cost allocation scheme that satisfies the five properties. Specifically, the Mertens mechanism $\Psi_{M}$ is given by

$$
\Psi_{M, i}^{|\mathcal{F}|}(r, \alpha) \triangleq \mathrm{E}\left[\int_{0}^{1} D_{\alpha * C, e_{i}} r(t \alpha) d t\right]
$$

where $e_{i} \in \mathbb{R}^{|\mathcal{F}|}$ is the $i$ th unit vector, the expectation is taken over a random vector $C \in \mathbb{R}^{|\mathcal{F}|}$ of independent standard Cauchy random variables, and

$$
D_{u} r(\alpha) \triangleq \lim _{\epsilon \rightarrow 0^{+}} \frac{r(\alpha+\epsilon u)-r(\alpha)}{\epsilon}, \quad D_{u, v} r(\alpha) \triangleq \lim _{\epsilon \rightarrow 0^{+}} \frac{D_{u+\epsilon v} r(\alpha)-D_{u} r(\alpha)}{\epsilon},
$$

are directional derivatives of $r(\alpha)$, given directions $u, v \in \mathbb{R}^{|\mathcal{F}|}$.

We propose the Mertens mechanism for the attribution rules in the context of piecewise linear systemic risk measures. The following lemma gives a sufficient condition for a homogeneous systemic risk measures $\rho$ to be piecewise linear. Recall that $\rho$ is generated by index sets $\mathcal{S}$ and $\mathcal{T}$ in Corollary 2 .

Lemma 2. Suppose index sets $\mathcal{S}$ and $\mathcal{T}$ are finite, $\Lambda^{(s)}$ is a piecewise linear continuous function for all $s \in \mathcal{S}$, and $\rho^{(t)}$ is a piecewise linear continuous function for all $t \in \mathcal{T}$. Then the homogeneous systemic risk measure $\rho$ generated by index sets $\mathcal{S}$ and $\mathcal{T}$ is piecewise linear and continuous.

Proof. It is immediate from the representation in Corollary 2.

Suppose the hypothesis of Lemma 2 is satisfied. Then, $r_{\rho}(\alpha)$ is clearly piecewise linear. Thus the Mertens mechanism can be used for risk attribution. Note that this attribution scheme does not have the 'no-undercut' property introduced in Theorem 4. Further, risk attribution for general non-differentiable systemic risk measures beyond this piecewise linear class is an area for future investigation.

\subsection{Examples}

In this section, we describe examples of homogeneous systemic risk measures.

Example $12(\mathrm{VaR})$. Consider the aggregation function $\Lambda_{\text {total }}(x) \triangleq \mathbf{1}_{\mathcal{F}}^{\top} x$ of (1), i.e., the total profit and loss across all firms. Given a probability distribution $p$ over the scenarios $\Omega$ and a confidence level $\zeta$, consider the value-at-risk function $\mathrm{VaR}_{\zeta}$ of (17) as a base risk measure. Then the homogeneous systemic risk measure

$$
\rho_{\mathrm{VaR}}(X) \triangleq\left(\operatorname{VaR}_{\zeta} \circ \Lambda_{\text {total }}\right)(X)=\operatorname{VaR}_{\zeta}\left(\mathbf{1}_{\mathcal{F}}^{\top} X_{1}, \ldots, \mathbf{1}_{\mathcal{F}}^{\top} X_{|\Omega|}\right)
$$

is simply the value-at-risk of the aggregated outcomes. Note that $\Lambda_{\text {total }}$ and $\mathrm{VaR}_{\zeta}$ are piecewise linear and continuous. 
When the probability measure $p$ over the scenarios is arbitrary, we can consider conditional probability measures of the form $\mathbb{P}(\omega \mid A)$ where $A \subset \Omega$ denotes a set of stress scenarios. This conditional variation of the value-at-risk is in the same spirit as the CoVaR measure defined by Adrian and Brunnermeier (2009). Unlike them, we only condition on subsets of the exogenously defined scenarios. This restriction is required in order to be able compare different sets of outcomes on the same set of scenarios.

In this example, we can also illustrate the Mertens mechanism for risk attribution. For ease of exposition, assume that the probability distribution over scenarios is given by the uniform distribution, i.e., $p \triangleq \mathbf{1}_{\Omega} /|\Omega|$. As in Example 11 , the risk $\rho_{\mathrm{VaR}}(X)$ takes the value $\left(1_{\mathcal{F}}^{\top} X\right)_{\left[k^{*}\right]}$, i.e., the $k^{*}$ th order statistic of the aggregated losses across all firms. Now, assume that this value is achieved by a unique scenario $\omega^{*}$, so that $\rho \mathrm{VaR}(X)=\mathbf{1}_{\mathcal{F}}^{\top} X_{\omega^{*}}$. Then, in fact, for all $t>0$, $r_{\mathrm{VaR}}\left(t \mathbf{1}_{\mathcal{F}}\right)=\rho_{\mathrm{VaR}}(t X)=t \mathbf{1}_{\mathcal{F}}^{\top} X_{\omega^{*}}$. Also, in this case, $r_{\mathrm{VaR}}$ will be a linear function in the neighborhood of each $\alpha=t \mathbf{1}_{\mathcal{F}}$. Thus, we have the directional derivatives

$$
D_{u} r_{\mathrm{VaR}}\left(t \mathbf{1}_{\mathcal{F}}\right)=u^{\top} X_{\omega^{*}}, \quad D_{u, e_{i}} r_{\mathrm{VaR}}\left(t \mathbf{1}_{\mathcal{F}}\right)=X_{i, \omega^{*}}
$$

for all directions $u$. Then, the risk attribution to firm $i$ according to the Mertens mechanism (19) simplifies to become

$$
\Psi_{M, i}^{|\mathcal{F}|}\left(r_{\mathrm{VaR}}, \mathbf{1}_{\mathcal{F}}\right)=X_{i, \omega^{*}}
$$

In other words, the risk attribution of firm $i$ will be the loss incurred by the firm in the critical scenario $\omega^{*}$. More generally, if the value-at-risk is achieved in multiple scenarios, the risk attribution for each firm will be an average of losses across these scenarios.

Example 13 (Comonotonic Risk Measures). Following Kou et al. (2009), one can defined homogeneous aggregation functions of the form

$$
\Lambda(x) \triangleq \sum_{k=1}^{|\mathcal{F}|} \gamma_{k} x_{[k]},
$$

where $\gamma \in \mathbb{R}_{+}^{|\mathcal{F}|}, \mathbf{1}_{\mathcal{F}}^{\top} \gamma=|\mathcal{F}|$, and $x_{[k]}$ denotes the $k$ th order statistic of the vector $x$. Note that the value-at-risk and the median are special cases of this risk function. Fix a probability measure $p$ over the scenarios. Combining this aggregation function with the homogeneous single-firm risk measure

$$
\rho_{0}(x) \triangleq p^{\top} x
$$

we get the homogeneous systemic risk measure

$$
\rho(X) \triangleq\left(\rho_{0} \circ \Lambda\right)(X)=\sum_{\omega \in \Omega} p_{\omega} \Lambda\left(X_{\omega}\right)
$$

Note that $\Lambda$ and $\rho_{0}$ are piecewise linear and continuous. Since all comonotonic risk measures are homogeneous risk measures, it follows that all our results in this section apply to such risk measures. 


\section{References}

V. V. Acharya, L. H. Pedersen, T. Philippon, and M. Richardson. Measuring systemic risk. Working paper, March 2010a.

V. V. Acharya, T. Yorulmazer, and J. A. C. Santos. Systemic risk and deposit insurance premiums. Federal Reserve Bank of New York Economic Policy Review, 16(1):89-99, August 2010b.

T. Adrian and M. Brunnermeier. CoVaR. Federal Reserve Bank of New York Staff Reports, 348:1-8, 2009.

P. Artzner, F. Delbaen, J.-M. Eber, and D. Heath. Coherent measures of risk. Mathematical Finance, 9(3): 203-228, July 1999.

R. J. Aumann and L. S. Shapley. Values of Non-Atomic Games. Princeton University Press, 1974.

D. P. Bertsekas. Nonlinear Programming. Athena Scientific, Belmont, MA, 2nd edition, 1999.

L. J. Billera and D. C. Heath. Allocation of shared costs: A set of axioms yielding a unique procedure. Mathematics of Operations Research, 7:32-39, 1982.

A. Buch and G. Dorfleitner. Coherent risk measures, coherent capital allocations and the gradient allocation principle. Insurance: Mathematics and Economics, 42(1):235-242, 2008. ISSN 0167-6687.

M. Castellani. A dual representation for proper positively homogeneous functions. Journal of Global Optimization, 16(4):393-400, 2000.

S. Cerreia-Vioglio, F. Maccheroni, M. Marinacci, and L. Montrucchio. Risk measures: Rationality and diversification. Mathematical Finance, 2010.

A. Cherny and D. Orlov. On two approaches to coherent risk contribution. Mathematical Finance, 21(3): $557-571,2011$.

R. Cont, A. Moussa, and E. Bastos e Santos. Network structure and systemic risk in banking systems. Working paper, December 2010.

M. Denault. Coherent allocation of risk capital. Journal of Risk, 4:1-34, 2001. ISSN 1465-1211.

L. Eisenberg and T. H. Noe. Systemic risk in financial systems. Management Science, 47(2):236-249, 2001.

T. Fischer. Risk capital allocation by coherent risk measures based on one-sided moments. Insurance: Mathematics and Economics, 32:135-146, 2003.

C. Gauthier, A. Lehar, and M. Souissi. Macroprudential regulation and systemic capital requirements. Technical Report 2010-4, Bank of Canada, January 2010.

K. Giesecke and B. Kim. Systemic risk: What defaults are telling us. Working paper, January 2011.

O. Haimanko. Cost sharing: the nondifferentiable case. Journal of Mathematical Economics, 35:445-462, 2001.

X. Huang, H. Zhou, and H. Zhu. A framework for assessing the systemic risk of major financial institutions. Journal of Banking 83 Finance, 33(11):2036-2049, 2009.

P. Jorion. Value at Risk. McGraw-Hill, New York, 2006.

S. G. Kou, X. Peng, and C. C. Heyde. External risk measures and Basel accords. Working paper, Columbia University and Hong Kong University of Science and Technology, 2009.

D. Kreps. Notes on the Theory of Choice. Westview Press, 1988. 
A. Lehar. Measuring systemic risk: A risk management approach. Journal of Banking 83 Finance, 29(10): 2577-2603, 2005.

M. Liu and J. Staum. Systemic risk components and deposit insurance premia. Technical Report 2011-03, Federal Deposit Insurance Corporation Center for Financial Research, December 2010.

J. F. Mertens. The Shapley value in the non-differentiable case. International Journal of Game Theory, 17: $1-65,1988$.

A. Schied. Risk measures and robust optimization problems. Stochastic Models, 22(4):753, 2006.

J. C. Staum. Systemic risk components and deposit insurance premia. Working paper, January 2011a.

J. C. Staum. Counterparty contagion in context: Contributions to systemic risk. Working paper, 2011b.

N. Tarashev, C. Borio, and K. Tsatsaronis. Attributing systemic risk to individual institutions. Technical Report 308, Working paper 308, Bank of International Settlements, May 2010.

A. Tsanakas. To split or not to split: Capital allocation with convex risk measures. Insurance: Mathematics and Economics, 44:268-277, 2009. 


\title{
Online Supplement to “An Axiomatic Approach To Systemic Risk"
}

\author{
Chen Chen \\ Industrial Engineering and Operations Research \\ Columbia University \\ email: cc3136@columbia.edu \\ Garud lyengar \\ Industrial Engineering and Operations Research \\ Columbia University \\ email: garud@ieor.columbia.edu \\ Ciamac C. Moallemi \\ Graduate School of Business \\ Columbia University \\ email: ciamac@gsb.columbia.edu
}

Initial Version: August 4, 2011

Current Revision: April 18, 2012

\section{A. Decentralized Implementation}

In this section, we explore the decentralization of systemic risk management. In particular, we establish that the dual representation of Section 3 and the risk attribution rule of Section 4 provide the basis of a tax scheme for internalizing the systemic risk into the decisions of individual firms.

To this end, for each firm $i \in \mathcal{F}$, let $\mathcal{T}^{(i)} \subset \mathbb{R}^{|\Omega|}$ denote the convex feasible set of possible outcomes for firm $i$ over the set of scenarios $\Omega$. The set $\mathcal{T}^{(i)}$ can be interpreted as the set of possible investment opportunities available to firm $i$. Denote by $\mathcal{T} \triangleq \mathcal{T}^{(1)} \times \mathcal{T}^{(2)} \times \ldots \times \mathcal{T}^{(|\mathcal{F}|)} \subset \mathbb{R}^{|\mathcal{F}| \times|\Omega|}$ the resulting set of possible economies. Let $U_{i}: \mathcal{T}^{(i)} \rightarrow \mathbb{R}$ denote the utility function of firm $i$. We assume that $U_{i}$ is strictly concave and differentiable. Given a systemic risk measure $\rho: \mathbb{R}^{|\mathcal{F}| \times|\Omega|} \rightarrow \mathbb{R}$, we make the following definition:

Definition 9 (Social Optimality). An economy $\bar{X}=\left(\bar{X}^{(1)} ; \bar{X}^{(2)} ; \ldots ; \bar{X}^{(|\mathcal{F}|)}\right) \in \mathcal{T}$ is socially optimal if it maximizes risk-adjusted welfare according to the optimization problem

$$
\bar{X} \in \underset{X \in \mathcal{T}}{\operatorname{argmax}}\left\{\sum_{i \in \mathcal{F}} U_{i}\left(X^{(i)}\right)-\tau \rho(X)\right\} .
$$

Here, $\tau>0$ is a parameter that captures the impact of the systemic risk externality.

A regulator or central planner wishes to drive individual firms to make decisions so that, collectively, these decisions results in a socially optimal economy. However, the regulator is not able to directly control the outcomes of each firm; it is only able to influence investment decisions indirectly via incentives. In particular, suppose the regulator imposes a tax $t_{i}\left(X^{(i)}\right)$ on firm $i$ given outcomes 
$X^{(i)} \in \mathcal{T}^{(i)}$. The firm would then choose outcomes so as to optimize its tax-adjusted utility, i.e., it would solve the optimization problem

$$
\underset{X^{(i)} \in \mathcal{T}^{(i)}}{\operatorname{maximize}}\left\{U_{i}\left(X^{(i)}\right)-t_{i}\left(X^{(i)}\right)\right\}
$$

Motivated by the dual representation of Section 3 and the risk attribution rule of Section 4 , the following theorem suggests a taxation scheme to implement any socially optimal economy:

Theorem 7. Suppose that $\bar{X} \in \mathcal{T}$ is a socially optimal economy. There exists $\Xi^{*} \in \mathbb{R}^{|\mathcal{F}| \times|\Omega|}$ that is an optimal solution to the dual problem (14) for the systemic risk $\rho(\bar{X})$ so that if we define, for each firm $i$, the tax function

$$
t_{i}\left(X^{(i)}\right) \triangleq \tau \sum_{\omega \in \Omega} \Xi_{i, \omega}^{*} X_{i, \omega}
$$

then, $\bar{X}^{(i)}$ is an optimal outcome for firm i, i.e.,

$$
\bar{X}^{(i)} \in \underset{X^{(i)} \in \mathcal{T}^{(i)}}{\operatorname{argmax}} U_{i}\left(X^{(i)}\right)-\tau \sum_{\omega \in \Omega} \Xi_{i, \omega}^{*} X_{i, \omega} .
$$

Proof. Since $\bar{X}$ is a socially optimal economy, first order conditions for optimality for A.1 imply that there must be a subgradient $\Xi^{*} \in \mathbb{R}^{|\mathcal{F}| \times|\Omega|}$ of $\rho$ at $\bar{X}$ so that

$$
\sum_{i \in \mathcal{F}} \sum_{\omega \in \Omega}\left(\nabla_{i, \omega} U_{i}\left(X^{(i)}\right)-\tau \Xi_{i, \omega}^{*}\right)\left(X_{i, \omega}-\bar{X}_{i \omega}\right) \leq 0
$$

for all $X \in \mathcal{T}$. Any subgradient $\Xi^{*}$ is clearly a dual optimal solution to (14), according to Danskin's theorem (Bertsekas, 1999).

Now, given $i \in \mathcal{F}$, we can take $X^{(j)}=\bar{X}^{(j)}$ for all $j \neq i$ in A.3. We obtain that, for all $i \in \mathcal{F}$,

$$
\sum_{\omega \in \Omega}\left(\nabla_{i, \omega} U_{i}\left(X^{(i)}\right)-\tau \Xi_{i, \omega}^{*}\right)\left(X_{i, \omega}-\bar{X}_{i \omega}\right) \leq 0
$$

for all $X^{(i)} \in \mathcal{T}^{(i)}$. Note that A.4 is the first order optimality condition for A.2), and thus $\bar{X}^{(i)}$ is an optimal solution to A.2.

Theorem 7 demonstrates that the objective of the regulator can be aligned with the incentives of individual firms through taxation schemes. Moreover, the tax paid by each firm is (up to the constant $\tau$ ) determined by the risk attribution of that firm, c.f. Definition 5 .

Since the taxation scheme in Theorem 7 is based on a subgradient $\Xi^{*}$ at the social optimal economy $\bar{X}$, it appears that, in order to compute this taxation scheme, the regulator would need to solve the centralized problem A.1. In other words, the regulator would need to know the utilities $U_{i}$ and investment opportunities $\mathcal{T}^{(i)}$ of each firm $i$. While it is reasonable to assume that the regulator knows the risk function $\rho$ and that it can demand that the firms reveal their losses $X$ across scenarios, it is unlikely that the firms would reveal their utility functions or investment 
opportunities. This obstacle can be overcome via an iterative scheme provided we assume that each $U_{i}$ is a smooth, strictly concave function, and that

(i) the firms are myopic, i.e., the optimize their loss profiles with the given taxation scheme without considering the impact of their decisions on future taxes, and

(ii) the firms report their loss profiles truthfully.

The second condition can be achieved in a regulatory regime with suitable penalties. The first can be justified on the basis of bounded rationality on the part of the firms. Consider the following scheme, in each iteration $k$ :

1. Each firm $i$ computes

$$
X_{k}^{(i)} \in \underset{X \in \mathcal{T}^{(i)}}{\operatorname{argmax}}\left\{U_{i}\left(X^{(i)}\right)-\tau\left(\Xi_{i}^{(k)}\right)^{\top}\left(X^{(i)}-X_{k-1}^{(i)}\right)-\frac{\nu_{k}}{2}\left\|X^{(i)}-X_{k-1}^{(i)}\right\|_{2}^{2}\right\},
$$

where $X_{k-1}^{(i)}$ denotes the loss profile of the firm in iteration prior $k-1$, and $\nu_{k}\left\|X^{(i)}-X_{k-1}^{(i)}\right\|_{2}^{2}$ is a proximal term that prevents large changes in $X^{(i)}$ in each iteration. The optimization problem above can be interpreted as a utility maximization problem with convex taxes.

2. The regulator receives the outputs $X_{k}=\left(X_{k}^{(i)}, \ldots, X_{k}^{(|\mathcal{F}|)}\right)$ and computes a subgradient $\Xi_{k}$ of $\rho$ at $X_{k}$.

There exists a suitable choice for the sequence of regularization parameters $\left\{\tau_{k}\right\}$ such that proximal subgradient scheme above converges to a socially optimal economy $\bar{X}$ and the subgradient $\Xi_{k}$ converge to a subgradient $\bar{\Xi}$ of $\rho$ at $\bar{X}$ such that the first order optimality condition (A.4) hold for the pair $(\bar{X}, \bar{\Xi})$ (e.g., Bertsekas, 1999). Note that each iteration of the above subgradient scheme, the regulator only requires the firms to truthfully communicate the current iterate $X_{k}$ and not their utility functions.

\section{B. Proofs}

Theorem 3. Suppose $\rho=\rho_{0} \circ \Lambda$ is a systemic risk measure characterized by an aggregation function $\Lambda$ and a base risk measure $\rho_{0}$. Then, for all economies $X \in \mathbb{R}^{|\mathcal{F}| \times|\Omega|}, \rho(X)$ can be expressed as the value of the optimization problem

$$
\begin{array}{lll}
\rho(X)=\underset{\bar{\pi}, \Xi}{\operatorname{maximize}} & \sum_{i \in \mathcal{F}} \sum_{\omega \in \Omega} \Xi_{i, \omega} X_{i, \omega} & \\
\text { subject to } & (1, \bar{\pi}) \in \mathcal{A}^{*}, & \\
& \left(\bar{\pi}_{\omega}, \Xi_{\omega}\right) \in \mathcal{B}^{*}, \quad \forall \omega \in \Omega, \\
& \bar{\pi} \in \mathbb{R}^{|\Omega|}, \Xi \in \mathbb{R}^{|\mathcal{F}| \times|\Omega|} . &
\end{array}
$$


In addition, feasible points $(\bar{\pi}, \Xi)$ for this problem must satisfy

$$
\bar{\pi} \geq \mathbf{0}_{\Omega}, \quad \mathbf{1}_{\Omega}^{\top} \bar{\pi} \leq 1, \quad \Xi \geq \mathbf{0}_{\mathcal{E}}, \quad \mathbf{1}_{\mathcal{F}}^{\top} \Xi \leq|\mathcal{F}| \bar{\pi}^{\top}
$$

Proof. In this proof, we use the primal representation of Theorem 2, Recall that primal representation of $\rho$ is

$$
\rho(X)=\inf _{(m, \ell) \in \mathbb{R} \times \mathbb{R}^{|\Omega|}} m+I_{\mathcal{A}}(m, \ell)+\sum_{\omega \in \Omega} I_{\mathcal{B}}\left(\ell_{\omega}, x_{\omega}\right),
$$

where $I_{\mathcal{A}}$ and $I_{\mathcal{B}}$ are indicator functions of sets $\mathcal{A}$ and $\mathcal{B}$ from 11 respectively. Here, given a set $\mathcal{C} \in \mathbb{R}^{n}$, we define the indicator function $I_{\mathcal{C}}: \mathbb{R}^{n} \rightarrow \mathbb{R} \cup\{\infty\}$ by

$$
I_{\mathcal{C}}(x) \triangleq \begin{cases}0 & \text { if } x \in \mathcal{C} \\ \infty & \text { otherwise }\end{cases}
$$

For the convex set $\mathcal{A}$, define the support function by

$$
S_{\mathcal{A}}\left(-\pi_{0}, \bar{\pi}\right) \triangleq \sup _{(m, \ell) \in \mathbb{R} \times \mathbb{R}|\Omega|}-\pi_{0} m+\bar{\pi}^{\top} \ell= \begin{cases}0 & \text { if }\left(\pi_{0}, \bar{\pi}\right) \in \mathcal{A}^{*} \\ \infty & \text { otherwise }\end{cases}
$$

where $\mathcal{A}^{*}$ is given by $(12)$. From convex duality, the indicator function of $\mathcal{A}$ can be expressed as the Fenchel-Legendre conjugate of its support function, i.e.,

$$
I_{\mathcal{A}}(m, \ell)=\sup _{\left(\pi_{0}, \bar{\pi}\right) \in \mathbb{R} \times \mathbb{R}|\Omega|}-\pi_{0} m+\bar{\pi}^{\top} \ell-S_{\mathcal{A}}\left(-\pi_{0}, \bar{\pi}\right)=\sup _{\left(\pi_{0}, \bar{\pi}\right) \in \mathcal{A}^{*}}-\pi_{0} m+\bar{\pi}^{\top} \ell
$$

Similarly, the indicator function for the set $\mathcal{B}$ can be expressed as

$$
I_{\mathcal{B}}\left(\ell_{\omega}, x_{\omega}\right)=\sup _{\left(\xi_{0 \omega}, \hat{\xi}_{\omega}\right) \in \mathcal{B}^{*}}-\xi_{0 \omega} \ell_{\omega}+\hat{\xi}_{\omega}^{\top} x_{\omega}
$$

where $\mathcal{B}^{*}$ is given by 13 .

Thus, applying convex duality to the primal representation, we obtain that

$$
\begin{aligned}
\rho(X)= & \inf _{(m, \ell) \in \mathbb{R} \times\left.\mathbb{R}\right|^{|\Omega|}} \sup _{\substack{\left(\pi_{0}, \bar{\pi}\right) \in \mathcal{A}^{*} \\
\left(\xi_{0 \omega}, \hat{\xi}_{\omega}\right) \in \mathcal{B}^{*}, \forall \omega \in \Omega}} m-\pi_{0} m+\bar{\pi}^{\top} \ell+\sum_{\omega \in \Omega}\left(-\xi_{0 \omega} \ell_{\omega}+\hat{\xi}_{\omega}^{\top} x_{\omega}\right) \\
= & \sup _{\substack{\left(\pi_{0}, \bar{\pi}\right) \in \mathcal{A}^{*} \\
\left(\xi_{0 \omega}, \hat{\xi}_{\omega}\right) \in \mathcal{B}^{*}, \forall \omega \in \Omega}} \inf _{(m, \ell) \in \mathbb{R} \times \mathbb{R}^{|\Omega|}} m-\pi_{0} m+\bar{\pi}^{\top} \ell+\sum_{\omega \in \Omega}\left(-\xi_{0 \omega} \ell_{\omega}+\hat{\xi}_{\omega}^{\top} x_{\omega}\right) \\
= & \sup _{\substack{(1, \bar{\pi}) \in \mathcal{A}^{*} \\
\left(\bar{\pi}_{\omega}, \hat{\xi}_{\omega}\right) \in \mathcal{B}^{*}, \forall \omega \in \Omega}} \sum_{\omega \in \Omega} \hat{\xi}_{\omega}^{\top} x_{\omega} .
\end{aligned}
$$

This establishes (B.1).

Now, we show the sub-stochastic properties $(B .2)$ for feasible dual variables $(\bar{\pi}, \Xi)$. Up to a sign 
change, $\mathcal{A}^{*}$ and $\mathcal{B}^{*}$ are the dual cones to $\mathcal{A}$ and $\mathcal{B}$, respectively. The monotonicity of the acceptance sets $\mathcal{A}$ and $\mathcal{B}$ implies that

$$
\bar{\pi} \geq \mathbf{0}_{\Omega}, \quad \Xi \geq \mathbf{0}_{\mathcal{E}} .
$$

From the normalization property of $\rho_{0}$, we have that $\left(1, \mathbf{1}_{\Omega}\right) \in \mathcal{A}$. This implies that

$$
1-\mathbf{1}_{\Omega}^{\top} \bar{\pi} \geq 0
$$

From the normalization property of $\Lambda$, we have that $\left(|\mathcal{F}|, \mathbf{1}_{\mathcal{F}}\right) \in \mathcal{B}$. This implies that

$$
|\mathcal{F}| \bar{\pi}_{\omega}-\mathbf{1}_{\mathcal{F}}^{\top} \Xi_{\omega} \geq 0, \quad \forall \omega \in \Omega
$$

Lemma 1. A function $g: \mathbb{R}^{n} \rightarrow \mathbb{R}$ is positively homogeneous and monotonic if and only if there exists an index set $\mathcal{S}$ where, for each $s \in \mathcal{S}, g^{(s)}: \mathbb{R}^{n} \rightarrow \mathbb{R} \cup\{\infty\}$ is a positively homogeneous, monotonic, and convex extended real-valued function, such that

$$
g(x)=\underset{s \in \mathcal{S}}{\operatorname{minimize}} g^{(s)}(x)
$$

for all $x \in \mathbb{R}^{n}$.

Proof. This proof closely follows the argument presented by Castellani (2000). Let $\mathcal{S} \triangleq\left\{x \in \mathbb{R}^{n}\right.$ : $\left.\|x\|_{2}=1\right\}$ denote the unit sphere in $\mathbb{R}^{n}$. For $s \in \mathcal{S}$, define

$$
\mathcal{T}_{s} \triangleq \mathcal{K}_{s}^{\circ}+g(s) s
$$

where $\mathcal{K}_{s} \triangleq\{\lambda s: \lambda \geq 0\}$ denotes the cone generated by $s \in \mathcal{S}$, and $\mathcal{K}_{s}^{\circ} \triangleq\left\{y: y^{\top} s \leq 0\right\}$ denotes the polar cone of $\mathcal{K}_{s}$. Given $s \in \mathcal{S}$, define

$$
g^{(s)}(x) \triangleq \sup _{y \in \mathcal{T}_{s} \cap \mathbb{R}_{+}^{n}} y^{\top} x
$$

Then $g^{(s)}: \mathbb{R}^{n} \rightarrow \mathbb{R} \cup\{\infty\}$ is clearly monotonic, positively homogeneous and convex. Using convex duality, we can rewrite $g^{(s)}$ as

$$
\begin{aligned}
g^{(s)}(x) & =\sup _{y \in \mathcal{T}_{s}, y \geq 0} y^{\top} x \\
& =\inf _{\beta \geq 0} \sup _{y \in \mathcal{T}_{s}} y^{\top}(x+\beta) \\
& =\inf _{\beta \geq 0}\left\{g(s) s^{\top}(x+\beta)+\inf _{z \in \mathcal{K}_{s}^{\circ}} z^{\top}(x+\beta)\right\} \\
& =\inf _{\beta \geq 0} g(s) s^{\top}(x+\beta)+I_{\mathcal{K}_{s}}(x+\beta),
\end{aligned}
$$

where $I_{\mathcal{K}_{s}}(z)$ denotes the indicator function $B .3$ for the set $\mathcal{K}_{s}$. The second equality follows from 
strong duality since $\mathcal{T}_{s}$ and $\mathbb{R}_{+}^{n}$ are non-empty polyhedral sets, and the last equality follows from the fact that, for all $z \in \mathbb{R}^{n}$,

$$
\sup _{y \in \mathcal{K}_{s}^{\circ}} y^{\top} z=I_{\mathcal{K}_{s}}(z)
$$

Since $\mathcal{S}$ is a compact set, for any $x \in \mathbb{R}^{n}, \inf _{s \in \mathcal{S}} g^{(s)}(x)$ is achieved, i.e., the infimum is, in fact, a minimum. Moreover,

$$
\begin{aligned}
\min _{s \in \mathcal{S}} g^{(s)}(x) & =\min _{s \in \mathcal{S}} \inf _{\beta \geq 0} g(s) s^{\top}(x+\beta)+I_{\mathcal{K}_{s}}(x+\beta) \\
& =\inf _{\beta \geq 0} \min _{s \in \mathcal{S}} g(s) s^{\top}(x+\beta)+I_{\mathcal{K}_{s}}(x+\beta) .
\end{aligned}
$$

Note that $I_{\mathcal{K}_{s}}(z)<\infty$ only if $z=\lambda s$, for some $\lambda \geq 0$. In that case, by positive homogeneity, $g(s) s^{\top} z+I_{\mathcal{K}_{s}}(z)=\lambda g(s)=g(\lambda s)=g(z)$. Thus, it follows that for all $z \in \mathbb{R}^{n}$,

$$
\min _{s \in \mathcal{S}} g(s) s^{\top} z+I_{\mathcal{K}_{s}}(z)=g(z) .
$$

Then, from (B.5), it follows that

$$
\min _{s \in \mathcal{S}} g^{(s)}(x)=\inf _{\beta \geq 0} g(x+\beta)=g(x)
$$

where the second equality follows from the fact that $g$ is monotonically increasing and $\beta \geq 0$. 